\title{
Characteristic polynomials for 1D random band matrices from the localization side
}

\author{
Mariya Shcherbina * Tatyana Shcherbina ${ }^{\dagger}$
}

\begin{abstract}
We study the special case of $n \times n$ 1D Gaussian Hermitian random band matrices, when the covariance of the elements is determined by $J=\left(-W^{2} \triangle+1\right)^{-1}$. Assuming that the band width $W \ll \sqrt{n}$, we prove that the limit of the normalized second mixed moment of characteristic polynomials (as $W, n \rightarrow \infty$ ) is equal to one, and so it does not coincides with those for GUE. This complements the result of 18 and proves the expected crossover for 1D Hermitian random band matrices at $W \sim \sqrt{n}$ on the level of characteristic polynomials.
\end{abstract}

\section{Introduction}

As in [18, we consider Hermitian $n \times n$ matrices $H_{n}$ whose entries $H_{i j}$ are random complex Gaussian variables with mean zero such that

$$
\mathbf{E}\left\{H_{i j} H_{l k}\right\}=\delta_{i k} \delta_{j l} J_{i j}
$$

where

$$
J_{i j}=\left(-W^{2} \Delta+1\right)_{i j}^{-1}
$$

and $\Delta$ is the discrete Laplacian on $\mathcal{L}=[1, n] \cap \mathbb{Z}$ with periodic boundary conditions. It is easy to see that the variance of matrix elements $J_{i j}$ is exponentially small when $|i-j| \gg W$, and so $W$ can be considered as the width of the band.

The density of states $\rho$ of the ensemble is given by the well-known Wigner semicircle law (see [3, 13] ):

$$
\rho(\lambda)=(2 \pi)^{-1} \sqrt{4-\lambda^{2}}, \quad \lambda \in[-2,2] .
$$

Random band matrices (RBM) are natural intermediate models to study eigenvalue statistics and quantum propagation in disordered systems, since they interpolate between mean-field type Wigner matrices (Hermitian or real symmetric matrices with i.i.d. random entries) and random Schrödinger operators, which have only a random diagonal potential in addition to the deterministic Laplacian on a box in $\mathbb{Z}^{d}$. In particular, RBM can be used to model the Anderson metal-insulator phase transition.

Let $\ell$ be the localization length, which describes the typical length scale of the eigenvectors of random matrices. The system is called delocalized if $\ell$ is comparable with the matrix size,

\footnotetext{
* Institute for Low Temperature Physics, Kharkiv, Ukraine, e-mail: shcherbina@ilt.kharkov.ua

${ }^{\dagger}$ School of Mathematics, Institute for Advanced Study, Princeton, USA, e-mail: tshcherbina@ias.edu. Supported by NSF grant DMS-1128155.
} 
and it is called localized otherwise. Delocalized systems correspond to electric conductors, and localized systems are insulators.

According to the physical conjecture (see [7, 12]) for 1D RBM the expected order of $\ell$ is $W^{2}$ (for the energy in the bulk of the spectrum), which means that varying $W$ we can see the crossover: for $W \gg \sqrt{n}$ the eigenvectors are expected to be delocalized, and for $W \ll \sqrt{n}$ they are localized.

The questions of the localization length are closely related to the universality conjecture of the bulk local regime of the random matrix theory. The bulk local regime deals with the behaviour of eigenvalues of $n \times n$ random matrices on the intervals whose length is of the order $O\left(n^{-1}\right)$. According to the Wigner - Dyson universality conjecture, this local behaviour does not depend on the matrix probability law (ensemble) and is determined only by the symmetry type of matrices. In this language the conjecture about the crossover for 1D RBM states that we get the same behaviour of eigenvalues correlation functions as for GUE (Hermitian matrices with i.i.d Gaussian entries) for $W \gg \sqrt{n}$ (which corresponds to delocalized states), and we get another behaviour determined by the Poisson statistics, for $W \ll \sqrt{n}$ (and corresponds to localized states).

At the present time only some upper and lower bounds for $\ell$ are proved rigorously. It is known from the paper [15] that $\ell \leq W^{8}$. On the other side, for the general Wigner matrices (i.e., $W=n$ ) the bulk universality has been proved in [11, 20], which gives $\ell \geq W$. By the developing the Erdős-Yau approach, there were also obtained some other results, where the localization length is controlled in a rather weak sense, i.e. the estimates hold for "most" eigenfunctions only: $\ell \geq W^{7 / 6}$ in [9] and $\ell \geq W^{5 / 4}$ in [10]. Gap universality for $W \sim n$ was proved very recently in [4].

Another method, which allows to work with random operators with non-trivial spatial structures, is supersymmetry techniques (SUSY) based on the representation of the determinant as an integral over the Grassmann variables. This method is widely used in the physics literature and is potentially very powerful but the rigorous control of the integral representations, which can be obtained by this method, is quite difficult. The rigorous application of SUSY to the Gaussian RBM which has the special block-band structure (special case of Wegner's orbital model) was developed in [19], where the universality of the bulk local regime for $W \sim n$ was proved. Combining this approach with Green's function comparison strategy it has been proved recently in [2] that $\ell \geq W^{7 / 6}$ (in a strong sense) for the block band matrices with rather general element's distribution. However, in the general case of RBM the question of bulk universality of local spectral statistics or of the order of the localization length is still open even for $d=1$.

Instead of eigenvalues correlation functions one can consider more simple objects which are the correlation functions of characteristic polynomials:

$$
F_{2 k}(\Lambda)=\mathbf{E}\left\{\prod_{s=1}^{2 k} \operatorname{det}\left(\lambda_{s}-H_{n}\right)\right\},
$$

where $\Lambda=\operatorname{diag}\left\{\lambda_{1}, \ldots, \lambda_{2 k}\right\}$ are real parameters that may depend on $n$. We are interested in the asymptotic behaviour of this function for

$$
\lambda_{j}=E+\frac{\xi_{j}}{n \rho(E)}, \quad E \in(-2,2) .
$$

From the SUSY point of view, correlation functions of characteristic polynomials correspond to the so-called fermion-fermion sector of the supersymmetric full model describing the usual 
correlation functions. They are especially convenient for the SUSY approach and were successfully studied by this techniques for many ensembles (see [5], [6], [16], [17, etc.). Although $F_{2 k}(\Lambda)$ is not a local object, it is also expected to be universal in some sense. Moreover, correlation functions of characteristic polynomials are expected to exhibit a crossover which is similar to that of local eigenvalue statistics. In particular, for 1D RBM they are expected to have the same local behaviour as for GUE for $W \gg \sqrt{n}$, and the different behaviour for $W \ll \sqrt{n}$. The first part of this conjecture was proved in [18]. The main result of [18] is

Theorem 1.1 ([18]) For the $1 D R B M$ of (1.1) - (1.2) with $W^{2}=n^{1+\theta}$, where $0<\theta \leq 1$, we have

$$
\lim _{n \rightarrow \infty} D_{2}^{-1} F_{2}\left(E+\frac{\xi}{n \rho(E)}, E-\frac{\xi}{n \rho(E)}\right)=\frac{\sin (2 \pi \xi)}{2 \pi \xi},
$$

i.e. coincides with those for GUE. The limit is uniform in $\xi$ varying in any compact set $C \subset \mathbb{R}$. Here $\rho(x)$ and $F_{2}$ are defined in (1.3) and (1.4), $E \in(-2,2)$, and

$$
D_{2}=F_{2}(E, E) \text {. }
$$

The purpose of the present paper is to study correlation functions of characteristic polynomials for (1.1) from the localization side $W \ll \sqrt{n}$ and to prove, that (1.6) is different in this case. The main result is

Theorem 1.2 For the $1 D R B M$ of (1.1) - (1.2) with $1 \ll W \leq \sqrt{n / C_{*} \log n}$ for sufficiently big $C_{*}$, we have

$$
\lim _{n \rightarrow \infty} D_{2}^{-1} F_{2}\left(E+\frac{\xi}{n \rho(E)}, E-\frac{\xi}{n \rho(E)}\right)=1,
$$

where the limit is uniform in $\xi$ varying in any compact set $C \subset \mathbb{R}$. Here $E \in(-2,2)$, and $\rho(x), F_{2}$, and $D_{2}$ are defined in (1.3), (1.4), and (1.7).

This theorem complements the previous one and proves the crossover of the bulk local regime of the random matrix theory on the level of correlation functions of characteristic polynomials.

Remark 1.1 Although the result is formulated for $\xi_{1}=-\xi_{2}=\xi$ in (1.5), one can prove Theorem 1.2 for $\xi_{1}, \xi_{2} \in C \subset \mathbb{R}$ by the same arguments with minor revisions. The only difference is a little bit more complicated expressions for $D_{2}$ and $K(\xi)$ (see 2.4 below).

To prove Theorem 1.2, we apply the transfer matrix approach to the integral representation obtained in [18] by the supersymmetry techniques (note that the integral representation does not contain Grassmann integrals, see Proposition 2.1). The main difficulty here is that the transfer operator $K(\xi)$, obtained from an integral representation (see (2.4) below), is not self-adjoint; thus perturbation theory is not easily applied in a rigorous way. One possible way to work with similar operators was suggested in [8], where the much simpler toy-version of $K(\xi)$ (not in the matrix space, and with one saddle point only) was studied. Here we propose another approach, which does not require the contour rotation.

The paper is organized as follows. In Section 2 we rewrite $F_{2}$ as a trace of the $n$-th degree of some transfer operator $K(\xi)$ (see (2.4) below) and reduce Theorem 1.2 to the statements on the top eigenvalues of the operator (see (2.6), (2.8)). These statements are proved in Section 4 (see Theorem 4.1). Section 3 deals with the most important preliminary results needed for Section 4. The proofs of some technical lemmas are given in Appendix. 


\section{Representation in the operator form}

As it was proved in [18], Lemma 1, we have

Proposition 2.1 ([18]) The second correlation function of the characteristic polynomials for $1 D$ Hermitian Gaussian band matrices, defined in (1.4), can be represented as follows:

$$
\begin{aligned}
& F_{2}\left(\Lambda_{0}+\frac{\hat{\xi}}{n \rho(E)}\right)=-\left(2 \pi^{2}\right)^{-n} \operatorname{det}^{-2} J \int \exp \left\{-\frac{W^{2}}{2} \sum_{j=1}^{n} \operatorname{Tr}\left(X_{j}-X_{j-1}\right)^{2}\right\} \\
& \times \exp \left\{-\frac{1}{2} \sum_{j=1}^{n} \operatorname{Tr}\left(X_{j}+\frac{i \Lambda_{0}}{2}+\frac{i \widehat{\xi}}{n \rho(E)}\right)^{2}\right\} \prod_{j=1}^{n} \operatorname{det}\left(X_{j}-i \Lambda_{0} / 2\right) \prod_{j=1}^{n} d X_{j},
\end{aligned}
$$

where $\hat{\xi}=\operatorname{diag}\{\xi,-\xi\}, \Lambda_{0}=E \mathbf{I}, X_{j} \in \operatorname{Herm}(2)$ (i.e., $2 \times 2$ Hermitian matrices), $X_{0}=X_{n}$, and

$$
d X_{j}=d\left(X_{j}\right)_{11} d\left(X_{j}\right)_{22} d \Re\left(X_{j}\right)_{12} d \Im\left(X_{j}\right)_{12} .
$$

Denote

$$
\mathcal{H}=L_{2}[\operatorname{Herm}(2)]
$$

Let $\mathcal{F}: \mathcal{H} \rightarrow \mathcal{H}, \mathcal{F}(\xi): \mathcal{H} \rightarrow \mathcal{H}$ be the operators of multiplication by

$$
\begin{aligned}
\mathcal{F}(X) & =\exp \left\{-\frac{1}{4} \operatorname{Tr}\left(X+\frac{i \Lambda_{0}}{2}\right)^{2}+\frac{1}{2} \operatorname{Tr} \log \left(X-i \Lambda_{0} / 2\right)-F_{*}\right\}, \\
\mathcal{F}_{\xi}(X) & =\mathcal{F}(X) \cdot \exp \left\{-\frac{i}{2 n \rho(E)} \operatorname{Tr} X \hat{\xi}\right\}
\end{aligned}
$$

respectively, where $F_{*}$ will be chosen below (see (3.3)). Let also $K, K(\xi): \mathcal{H} \rightarrow \mathcal{H}$ be the operators with the kernels

$$
\begin{aligned}
K(X, Y) & =\frac{W^{4}}{2 \pi^{2}} \mathcal{F}(X) \exp \left\{-\frac{W^{2}}{2} \operatorname{Tr}(X-Y)^{2}\right\} \mathcal{F}(Y) \\
K_{\xi}(X, Y) & =\frac{W^{4}}{2 \pi^{2}} \mathcal{F}_{\xi}(X) \exp \left\{-\frac{W^{2}}{2} \operatorname{Tr}(X-Y)^{2}\right\} \mathcal{F}_{\xi}(Y) .
\end{aligned}
$$

Define

$$
C_{n}(\xi)=\exp \left\{2 n F_{*}+\xi^{2} / n \rho(E)^{2}\right\}
$$

Then Proposition 2.1 can be reformulated as

$$
F_{2}\left(\Lambda_{0}+\frac{\hat{\xi}}{n \rho(E)}\right)=-C_{n}(\xi) \cdot W^{-4 n} \operatorname{det}^{-2} J \cdot \operatorname{Tr} K^{n}(\xi)
$$

and thus we are interested in the asymptotic behaviour of $\operatorname{Tr} K^{n}(\xi)$.

For arbitrary compact operator $M$ we denote $\lambda_{j}(M)$ the $j$ th (by its modulo) eigenvalue of $M$, so that $\left|\lambda_{0}(M)\right| \geq\left|\lambda_{1}(M)\right| \geq \ldots$

Assume that we have proved that

$$
\left|\frac{\lambda_{1}(K(\xi))}{\lambda_{0}(K(\xi))}\right| \leq e^{-C_{1} / W^{2}}, \quad\left|\lambda_{0}(K(\xi))\right|=1-C_{2} / W+O\left(W^{-2}\right) .
$$


Then

$$
\operatorname{Tr} K^{n}(\xi)=\lambda_{0}^{n}(K(\xi))(1+r)
$$

where

$$
\begin{gathered}
|r|=\left|\sum_{j=1}^{\infty}\left(\lambda_{j}(K(\xi)) / \lambda_{0}(K(\xi))\right)^{n}\right| \leq\left|\frac{\lambda_{1}(K(\xi))}{\lambda_{0}(K(\xi))}\right|^{n-2} \sum_{j=0}^{\infty}\left|\lambda_{j}(K(\xi))\right|^{2} \\
\leq C e^{-C n / W^{2}} \int\left|K_{\xi}(X, Y)\right|^{2} d X d Y \leq C W^{4} e^{-C C_{*} \log n}=o(1), \quad W \rightarrow \infty .
\end{gathered}
$$

Similarly,

$$
D_{2}=-C_{n}(0) \cdot W^{-4 n} \operatorname{det}^{-2} J \cdot \lambda_{0}^{n}(K(0)) \cdot(1+o(1))
$$

Thus, the assertion of Theorem 1.2 follows from (2.5) and (2.7) combined with the relation

$$
\left|\lambda_{0}(K(\xi))-\lambda_{0}(K(0))\right|=o\left(n^{-1}\right), \quad n \rightarrow \infty .
$$

\section{Preliminary results}

To prove (2.6), consider stationary points of the function $\mathcal{F}$ of (2.2). It is easy to see that they are

$$
\begin{aligned}
X_{+} & =a_{+} \mathbf{I}, \quad X_{-}=a_{-} \mathbf{I} \\
X_{ \pm}(U) & =a_{+} U L U^{*}, \quad U \in \stackrel{\circ}{U}(2),
\end{aligned}
$$

where $\stackrel{\circ}{U}(2)=U(2) / U(1) \times U(1)$,

$$
a_{+}=-a_{-}=\sqrt{1-E^{2} / 4}, \quad L=\left(\begin{array}{cc}
1 & 0 \\
0 & -1
\end{array}\right) .
$$

Choose now $F_{*}$ of $(2.2)$ as

$$
F_{*}=\frac{1}{4} \operatorname{Tr}\left(a_{+} I+\frac{i \Lambda_{0}}{2}\right)^{2}-\frac{1}{2} \operatorname{Tr} \log \left(a_{+} I-i \Lambda_{0} / 2\right) .
$$

Then the value of $|\mathcal{F}|$ at points (3.1) is 1 .

Put

$$
X=\left(\begin{array}{cc}
a_{1} & \left(x_{1}+i y_{1}\right) / \sqrt{2} \\
\left(x_{1}-i y_{1}\right) / \sqrt{2} & b_{1}
\end{array}\right), \quad Y=\left(\begin{array}{cc}
a_{2} & \left(x_{2}+i y_{2}\right) / \sqrt{2} \\
\left(x_{2}-i y_{2}\right) / \sqrt{2} & b_{2}
\end{array}\right) .
$$

Rewrite $K(X, Y), K_{\xi}(X, Y)$ as

$$
\begin{aligned}
& K_{\xi}(X, Y)=K(X, Y)+\widetilde{K}(X, Y) \\
& K(X, Y)=A\left(a_{1}, a_{2}\right) A\left(b_{1}, b_{2}\right) A_{1}(X, Y),
\end{aligned}
$$


where the kernels $A$ and $A_{1}$ have the form

$$
\begin{aligned}
& A(x, y)=F(x) B(x, y) F(y), \quad B(x, y)=(2 \pi)^{-1 / 2} W e^{-W^{2}(x-y)^{2} / 2} ; \\
& F(x)=e^{-f(x) / 2}, \quad f(x)=(x+i E / 2)^{2} / 2-\log (x-i E / 2)-F_{*} \\
& A_{1}(X, Y)=F_{1}(X) B\left(x_{1}, x_{2}\right) B\left(y_{1}, y_{2}\right) F_{1}(Y) \\
& F_{1}(X)=\exp \left\{-\frac{1}{4}\left(x_{1}^{2}+y_{1}^{2}\right)+\frac{1}{2} \log \left(1-\frac{x_{1}^{2}+y_{1}^{2}}{2\left(a_{1}-i E / 2\right)\left(b_{1}-i E / 2\right)}\right)\right\}
\end{aligned}
$$

and the perturbation kernel $\widetilde{K}$ is

$$
\widetilde{K}(X, Y)=A\left(a_{1}, a_{2}\right) A\left(b_{1}, b_{2}\right) A_{1}(X, Y)\left(e^{-\frac{i}{2 n \rho(E)}\left(\xi\left(a_{1}-b_{1}\right)+\xi\left(a_{2}-b_{2}\right)\right)}-1\right) .
$$

Note that

$$
\begin{aligned}
& f\left(a_{+}\right)=\Re f\left(a_{-}\right)=f^{\prime}\left(a_{+}\right)=f^{\prime}\left(a_{-}\right)=0, \quad f\left(a_{ \pm}+x\right)-f\left(a_{ \pm}\right)=c_{ \pm} x^{2}+c_{3 \pm} x^{3}+\ldots ; \\
& c_{ \pm}=a_{+}\left(\sqrt{4-E^{2}} \pm i E\right) / 2, \quad \Re c_{+}=\Re c_{-}>0 ; \\
& \|B\| \leq 1, \quad\|A\| \leq 1 .
\end{aligned}
$$

Another representation of $K(X, Y), \widetilde{K}(X, Y)$ can be obtained by using polar coordinates. Namely, changing the variables

$$
X=U \Lambda U^{*}, \quad \Lambda=\operatorname{diag}\{a, b\}, \quad a>b, \quad U \in \stackrel{\circ}{U}(2),
$$

we obtain that $K(\xi)=K+\widetilde{K}$ can be represented as an integral operator in $L_{2}\left[\mathbb{R}^{2}, p\right] \times$ $L_{2}[\stackrel{\circ}{U}(2), d U]$ defined by the kernel

$$
K_{\xi}(X, Y)=K\left(a_{1}, a_{2}, b_{1}, b_{2}, U_{1}, U_{2}\right)+\widetilde{K}\left(a_{1}, a_{2}, b_{1}, b_{2}, U_{1}, U_{2}\right),
$$

where

$$
\begin{aligned}
& K\left(a_{1}, a_{2}, b_{1}, b_{2}, U_{1}, U_{2}\right)=t^{-1} A\left(a_{1}, a_{2}\right) A\left(b_{1}, b_{2}\right) K_{*}\left(t, U_{1}, U_{2}\right) ; \\
& K_{*}\left(t, U_{1}, U_{2}\right):=W^{2} t \cdot e^{t W^{2} \operatorname{Tr} U_{1} U_{2}^{*} L\left(U_{1} U_{2}^{*}\right)^{*} L / 4-t W^{2} / 2} ; \\
& \widetilde{K}\left(a_{1}, a_{2}, b_{1}, b_{2}, U_{1}, U_{2}\right)=K\left(a_{1}, a_{2}, b_{1}, b_{2}, U_{1}, U_{2}\right)\left(e^{\left(\nu\left(a_{1}, b_{1}, U_{1}\right)+\nu\left(a_{2}, b_{2}, U_{2}\right)\right) / n}-1\right) ; \\
& \nu(a, b, U)=-\frac{i \xi(a-b)}{4 \rho(E)} \operatorname{Tr} U L U^{*} L .
\end{aligned}
$$

Here and everywhere below

$$
t=\left(a_{1}-b_{1}\right)\left(a_{2}-b_{2}\right), \quad p(a, b)=\frac{\pi}{2}(a-b)^{2}
$$

and we denote by $d U$ the integration with respect the Haar measure on the group $\stackrel{U}{U}(2)$. The scalar product and the action of an integral operator in $L_{2}\left[\mathbb{R}^{2}, p\right] \times L_{2}[\stackrel{\circ}{U}(2), d U]$ are

$$
\begin{aligned}
& (f, g)_{p}=\int f(a, b) \bar{g}(a, b) p(a, b) d a d b, \quad p(a, b)=\frac{\pi}{2}(a-b)^{2} \\
& (M f)\left(a_{1}, b_{1}, U_{1}\right)=\int M\left(a_{1}, a_{2}, b_{1}, b_{2}, U_{1}, U_{2}\right) f\left(a_{2}, b_{2}, U_{2}\right) p\left(a_{2}, b_{2}\right) d a_{2} d b_{2} d U_{2} .
\end{aligned}
$$

Now let us study the operators $A$ and $K_{*}$ appearing in (3.5) and (3.10) 


\subsection{Analysis of the operator $A$}

Theorem 3.1 Operator $A$ of (3.5) has exactly one eigenvalue in each of the $C W^{-3 / 2}$. neighbourhoods of $\lambda_{0,+}$ and $\lambda_{0,-}$, where

$$
\begin{aligned}
& \lambda_{0, \pm}=\left(1+\frac{2 \alpha_{ \pm}}{W}+\frac{c_{ \pm}}{W^{2}}\right)^{-1 / 2} \\
& \alpha_{ \pm}=\sqrt{\frac{c_{ \pm}}{2}}\left(1+\frac{c_{ \pm}}{2 W^{2}}\right)^{1 / 2}
\end{aligned}
$$

Moreover, $\left|\lambda_{2}(A)\right| \leq\left|\lambda_{0,+}\right|-c_{1} / W$ with some absolute $c_{1}>0$.

The proof of the theorem is based on the proposition, which is the standard linear algebra tool

Proposition 3.1 Given a compact operator $\mathcal{K}$, assume that there is an orthonormal basis $\left\{\Psi_{l}\right\}_{l \geq 0}$ such that the resolvent

$$
\widehat{\mathcal{G}}_{j k}(z)=(\widehat{\mathcal{K}}-z)_{j k}^{-1}, \quad \widehat{\mathcal{K}}=\left\{\mathcal{K}_{j k}\right\}_{j, k=1}^{\infty}
$$

is uniformly bounded in $z \in \Omega \subset \mathbb{C}$, where $\Omega$ is some domain. Then the eigenvalues of $\mathcal{K}$ in $\Omega$ coincide with zeros of the function

$$
\begin{aligned}
F(z) & :=\mathcal{K}_{00}-z-\left(\widehat{\mathcal{G}}(z) \kappa, \kappa^{*}\right) \\
\kappa & =\left(\mathcal{K}_{10}, \mathcal{K}_{20}, \ldots\right), \kappa^{*}=\left(\mathcal{K}_{10}^{*}, \mathcal{K}_{20}^{*}, \ldots\right)
\end{aligned}
$$

The proof of the proposition follows from the standard Schur inversion formula

$$
\begin{aligned}
& \mathcal{G}_{i j}(z)=\widehat{\mathcal{G}}_{i j}(z)+(\widehat{\mathcal{G}} \kappa)_{i}\left(\widehat{\mathcal{G}} \kappa^{*}\right)_{j} / F(z), \quad i \neq 0, j \neq 0, \\
& \mathcal{G}_{0 j}(z)=-\left(\widehat{\mathcal{G}} \kappa^{*}\right)_{j} / F(z), \quad \mathcal{G}_{i 0}(z)=-(\widehat{\mathcal{G}} \kappa)_{i} / F(z), \quad \mathcal{G}_{00}(z)=(F(z))^{-1},
\end{aligned}
$$

valid for any $z: F(z) \neq 0$.

\subsubsection{Proof of Theorem 3.1}

To apply the proposition, let us first introduce and study the "model" operator

$$
A_{*}^{\left(c_{*}\right)}(x, y)=\mathcal{F}_{*}(x) B(x, y) \mathcal{F}_{*}(y), \quad \mathcal{F}_{*}(x)=e^{-c_{*} x^{2} / 2}, \quad \Re c_{*}>0 .
$$

Take

$$
\alpha=\sqrt{\frac{c_{*}}{2}}\left(1+\frac{c_{*}}{2 W^{2}}\right)^{1 / 2}=: \alpha_{1}+i \alpha_{2},
$$

and consider the system of functions

$$
\begin{aligned}
& \psi_{0}(x)=e^{-\alpha W x^{2}} \sqrt[4]{\alpha W / \pi} \\
& \psi_{k}(x)=h_{k}^{-1 / 2} e^{-\alpha W x^{2}} e^{2 \alpha_{1} W x^{2}}\left(\frac{d}{d x}\right)^{k} e^{-2 \alpha_{1} W x^{2}},=e^{-\alpha W x^{2}} p_{k}(x) \\
& h_{k}=k !\left(4 \alpha_{1} W\right)^{k-1 / 2} \sqrt{2 \pi}, \quad k=1,2, \ldots
\end{aligned}
$$

It is easy to see that $\left\{p_{k}\right\}_{k=0}^{\infty}$ are polynomials, orthogonal with the weight $e^{-2 \alpha_{1} W x^{2}}$ ( $p_{k}$ is the $k$ th Hermite polynomial of $x \sqrt{2 \alpha_{1} W}$ with a proper normalization). 
Lemma $3.1\left\{\psi_{k}\right\}_{k \geq 0}$ is a orthonormal system in $L_{2}(\mathbb{R})$ and

$$
A_{*}^{\left(c_{*}\right)} \psi_{0}=\lambda_{0}^{\left(c_{*}\right)} \psi_{0}, \quad \lambda_{0}^{\left(c_{*}\right)}=\left(1+\frac{2 \alpha}{W}+\frac{c_{*}}{W^{2}}\right)^{-1 / 2},
$$

The matrix $A_{* j k}^{\left(c_{*}\right)}:=\left(A_{*}^{\left(c_{*}\right)} \psi_{k}, \psi_{j}\right)$ is upper triangular, $\left(A_{*}^{\left(c_{*}\right)}\right)_{j k}=0$ if $j, k$ have different evenness, and

$$
\begin{aligned}
& A_{* k k}^{\left(c_{*}\right)}=\left(\lambda_{0}^{\left(c_{*}\right)}\right)^{2 k+1}, \quad A_{* k, k+2}^{\left(c_{*}\right)}=-2 i \alpha_{2} \frac{\sqrt{(k+1)(k+2)}}{W}\left(1+O\left(\frac{k+1}{W}\right)\right), \\
& \left|A_{* k, k+2 p}^{\left(c_{*}\right)}\right| \leq \frac{C^{p}(k+1)^{p}}{W^{p}} .
\end{aligned}
$$

In addition, if $\left\{\widetilde{\psi}_{k}\right\}$ are defined by $(\overline{3.17})$ with $c_{*}$ replaced by some $c_{0}>0, \tilde{P}_{l}$ is a projection on the space, spanned on $\left\{\tilde{\psi}_{r}\right\}_{k=0}^{l}$, and $P_{m}$ is a similar projection for $\left\{\psi_{k}\right\}_{k=0}^{m}$, then for any $l, m>2$

$$
\left\|\tilde{P}_{l}\left(1-P_{m}\right)\right\| \leq C l^{3} / m
$$

with $C$, depending only on $c_{*}$ and $c_{0}$.

The proof of Lemma 3.1 is given in Appendix.

Choose $W, n$-independent $\delta>0$, which is small enough to provide that the domain $\omega_{\delta}=$ $\{x \in \mathbb{R}:|F(x)|>1-\delta\}$ contains two non intersecting sub domains $\omega_{\delta}^{+}, \omega_{\delta}^{-}$, such that each of $\omega_{\delta}^{+}, \omega_{\delta}^{-}$contains one of the points $x=a_{+}$and $x=a_{-}$of maximum $F(x)$ (easier speaking, $\omega_{\delta}^{+}, \omega_{\delta}^{-}$are two non-intersecting neighbourhood of points $a_{+}$and $\left.a_{-}\right)$.

Consider the basis $\left\{\psi_{k, \delta}^{+}\right\}$, obtained by the Gramm-Schmidt orthonormalization procedure of

$$
\psi_{k}^{+}(x)=\psi_{k}\left(x-a_{+}\right)
$$

on $\omega_{\delta}^{+}$. Here we take $\left\{\psi_{k}\right\}_{k=0}^{\infty}$ of (3.17) with $c_{*}=c_{+}$of (3.7). Since $\psi_{k, \delta}^{+}(x)=O\left(e^{-c W}\right)$ for $x \notin \omega_{\delta}^{+}$, one can obtain easily that

$$
\psi_{k, \delta}^{+}(x)=\psi_{k}^{+}(x)+O\left(e^{-c W}\right), \quad k \ll W .
$$

By the same way we construct $\left\{\psi_{k}^{-}(x)\right\}_{k=0}^{\infty}$ and $\left\{\psi_{k, \delta}^{-}(x)\right\}_{k=0}^{\infty}$ on $\omega_{\delta}^{-}$(with $c_{*}=c_{-}$). Take some sufficiently large but $W, n$-independent $m$ and denote $P_{+}$and $P_{-}$the projections on the subspaces spanned on the systems $\left\{\psi_{k, \delta}^{+}\right\}_{k=0}^{m}$ and $\left\{\psi_{k, \delta}^{-}\right\}_{k=0}^{m}$ respectively. Evidently these projection operators are orthogonal to each other. Set

$$
P=P_{+}+P_{-}, \quad \mathcal{L}_{1}=P L_{2}[\mathbb{R}], \quad \mathcal{L}_{2}=(1-P) L_{2}[\mathbb{R}], \quad L_{2}[\mathbb{R}]=\mathcal{L}_{1} \oplus \mathcal{L}_{2} .
$$

In order to apply Proposition 3.1 to $A$, we consider the operator $A$ as a block operator with respect to the decomposition (3.22). It has the form

$$
\begin{aligned}
& A^{(11)}=A_{+}^{(m)}+A_{-}^{(m)}+O\left(e^{-c W}\right), \quad A_{+}^{(m)}:=P_{+} A P_{+}, \quad A_{-}^{(m)}:=P_{-} A P_{-} ; \\
& A^{(12)}=P_{+} A\left(I_{+}-P_{+}\right)+P_{-} A\left(I_{-}-P_{-}\right)+O\left(e^{-c W}\right) ; \\
& A^{(21)}=\left(I_{+}-P_{+}\right) A P_{+}+\left(I_{-}-P_{-}\right) A P_{-}+O\left(e^{-c W}\right) ; \\
& A^{(22)}=(1-P) A(1-P),
\end{aligned}
$$


where $I_{+}$and $I_{-}$are the operators of multiplication by $1_{\omega_{\delta}^{+}}$and $1_{\omega_{\delta}^{-}}$. Indeed, since

$$
A \psi_{k, \delta}^{+}(x)=\int A(x, y) \psi_{k, \delta}^{+}(y) d y=\int \mathcal{F}(x) e^{-W^{2}(x-y)^{2} / 2} \mathcal{F}(y) \psi_{k, \delta}^{+}(y) d y
$$

$|\mathcal{F}(x)| \leq 1$, and evidently for $k \leq m \psi_{k, \delta}^{+}(y)$ is $O\left(e^{-c W}\right)$ for $\left|y-a_{+}\right| \geq \varepsilon$ with any small $W$-independent $\varepsilon$, we get

$$
A \psi_{k, \delta}^{+}(x)=O\left(e^{-c W}\right), \quad\left|x-a_{+}\right| \geq 2 \varepsilon .
$$

Therefore, for instance, $\left(P_{-} A P_{+}\right) f=O\left(e^{-c W}\right),\left(I_{-}-P_{-}\right) A P_{+}=O\left(e^{-c W}\right)$, etc., which gives (3.23).

Now let $\widehat{A}$ be the matrix $A$ without the row and the column corresponding to $\psi_{0, \delta}^{+}$, and $\widehat{A}^{(11)}, \widehat{A}^{(12)}$, and $\widehat{A}^{(21)}$ be the blocks of this matrix similar to (3.23). Denote also

$$
\begin{gathered}
a=A \psi_{0, \delta}^{+}-\left(A \psi_{0, \delta}^{+}, \psi_{0, \delta}^{+}\right) \psi_{0, \delta}^{+} \\
a^{*}=A^{*} \psi_{0, \delta}^{+}-\left(A^{*} \psi_{0, \delta}^{+}, \psi_{0, \delta}^{+}\right) \psi_{0, \delta}^{+}
\end{gathered}
$$

and set

$$
F(z)=\left(A \psi_{0, \delta}^{+}, \psi_{0, \delta}^{+}\right)-z-\left((\widehat{A}-z)^{-1} a, a^{*}\right)
$$

Then, according to Proposition 3.1, to prove Theorem 3.1, it suffices to show the bounds

$$
\begin{aligned}
& \|a\| \leq C W^{-3 / 2}, \quad\left\|a^{*}\right\| \leq C W^{-1} \\
& \left\|(\widehat{A}-z)^{-1}\right\| \leq C W, \quad\left(A \psi_{0, \delta}^{+}, \psi_{0, \delta}^{+}\right)=\lambda_{0,+}+O\left(W^{-3 / 2}\right)
\end{aligned}
$$

for $z$, satisfying the conditions

$$
1-\frac{3 \alpha_{1}}{2 W} \leq|z| \leq 1, \quad\left|z-\lambda_{0,-}\right|>C / W .
$$

Indeed, consider $z \in \omega_{+}=\left\{z:\left|z-\lambda_{0,+}\right| \leq C_{0} W^{-3 / 2}\right\}$ with sufficiently big $C_{0}$, and set

$$
F_{0}(z)=\lambda_{0,+}-z
$$

Then (3.25) implies

$$
\begin{aligned}
\left|F(z)-F_{0}(z)\right|=\mid\left(A \psi_{0, \delta}^{+}, \psi_{0, \delta}^{+}\right)-\lambda_{0,+} & -\left((\widehat{A}-z)^{-1} a, a^{*}\right) \mid \\
& \leq O\left(W^{-3 / 2}\right)<C_{0} W^{-3 / 2}=\left|F_{0}(z)\right|, \quad z \in \partial \omega_{+}
\end{aligned}
$$

for sufficiently big $C_{0}$. Since both functions are analytic in $\omega_{+}$, the Rouchet theorem gives that $F$ and $F_{0}$ have the same numbers of roots (i.e., one) in $\omega_{+}$. This yields the first assertion of Theorem 3.1 (for $\lambda_{0,-}$ the proof is the same). To prove the second assertion, consider any point $z_{0}$ outside of $\omega_{+}$satisfying (3.26) and take $\omega_{0}=\left\{z:\left|z-z_{0}\right| \leq C_{0} W^{-3 / 2}\right\}$. Then (3.27) is still true on $\partial \omega_{0}$, and hence the number of roots of $F$ in $\omega_{0}$ is the same as for $F_{0}$, i.e. zero. Therefore, $A$ has only one eigenvalue in the domain (3.26). Applying similar argument for $\lambda_{0,-}$ instead of $\lambda_{0,+}$, we obtain Theorem 3.1 with $c_{1}=\alpha_{1} / 2$.

Hence, we are left to prove (3.25). The bound $\left\|(\widehat{A}-z)^{-1}\right\| \leq C W$ follows from three lemmas. 
Lemma 3.2 Given z satisfying (3.26), we have

$$
\left\|\left(\widehat{A}^{(11)}-z\right)^{-1}\right\| \leq C W
$$

Lemma 3.3 We have

$$
\begin{aligned}
& \left\|A^{(12)}\right\| \leq C W^{-1},\left\|A^{(21)}\right\| \leq C W^{-3 / 2} \\
& \left\|a^{*}\right\| \leq C W^{-1},\|a\| \leq C W^{-3 / 2}
\end{aligned}
$$

Lemma 3.4 Given z satisfying (3.26), we have

$$
\left\|A^{(22)}\right\| \leq 1-C m^{1 / 3} / W, \quad \Rightarrow \quad\left\|\left(A^{(22)}-z\right)^{-1}\right\| \leq C W .
$$

\subsubsection{Proof of Lemmas $3.2-3.4$}

Proof of Lemma 3.2. According to (3.23), we have to prove that

$$
\left\|\left(\widehat{A}_{+}^{(m)}-z\right)^{-1}\right\| \leq C W, \quad\left\|\left(A_{-}^{(m)}-z\right)^{-1}\right\| \leq C W,
$$

where $\widehat{A}_{+}^{(m)}$ is $A_{+}^{(m)}$ without the line and the column, corresponding to $\psi_{0, \delta}^{+}$.

Let us prove the first inequality of (3.28).

Using that $\psi_{k, \delta}^{+}(y)$ is $O\left(e^{-c \log ^{2} W}\right)$ for $\left|y-a_{+}\right| \geq W^{-1 / 2} \log W$ for $k \leq m$, we get similarly to $(3.24)$

$$
A \psi_{k, \delta}^{+}(x)=O\left(e^{-c \log ^{2} W}\right), \quad\left|x-a_{+}\right| \geq 2 W^{-1 / 2} \log W .
$$

In addition, $A \psi_{k, \delta}^{+}(x)$ can be written in the form $(k=0,1, \ldots, m)$

$$
A \psi_{k, \delta}^{+}(x)=\int_{\left|y-a_{+}\right| \leq W^{-1 / 2} \log W}\left(A_{*}^{+}\left(x-a_{+}, y-a_{+}\right)+\widetilde{A}(x, y)\right) \psi_{k, \delta}^{+}(y) d y+O\left(e^{-c \log ^{2} W}\right) .
$$

Here and below we denote

$$
A_{*}^{ \pm}:=A_{*}^{\left(c_{ \pm}\right)}
$$

and

$$
\widetilde{A}(x, y)=A(x, y)-A_{*}^{+}\left(x-a_{+}, y-a_{+}\right) .
$$

Expanding $\mathcal{F}$ for $\left|x-a_{+}\right| \leq 2 W^{-1 / 2} \log W,\left|y-a_{+}\right| \leq 2 W^{-1 / 2} \log W$, we get

$$
\widetilde{A}(x, y)=A(x, y) O\left(W^{-3 / 2} \log ^{3} W\right), \quad \text { if }\left|x-a_{+}\right|+\left|y-a_{+}\right| \leq W^{-1 / 2} \log W .
$$

Thus, for $k \leq m$

$$
\widehat{A}_{+}^{(m)}=\widehat{A}_{+, *}^{(m)}+\widetilde{A}^{(11)} \text {, where } \widehat{A}_{+, *}^{(m)}=\left\{A_{* j k}^{+}\right\}_{j, k=1}^{m} \text {, and }\left\|\widetilde{A}^{(11)}\right\| \leq C W^{-3 / 2} .
$$

Hence it suffices to prove that for $z$ satisfying (3.26) we have

$$
\left\|\left(\widehat{A}_{+, *}^{(m)}-z\right)^{-1}\right\| \leq C W .
$$

Decompose

$$
\widehat{A}_{+, *}^{(m)}-z=D(z)+R, \quad \text { where } \quad D_{j k}=\delta_{j k}\left(A_{* k k}^{+}-z\right)
$$


By using (3.19) we get that $\left\|D(z)^{-1}\right\| \leq C W$ and for $j-k \neq 2$ all non-zero entries $\left(R D^{-1}\right)_{k j}=$ $O\left(W^{-1}\right)$ or less. In addition, according to (3.26)

$$
\begin{aligned}
& \left|D_{k k}\right|=\left|A_{* k k}^{+}-z\right|=\left|1-\frac{(2 k+1) \alpha_{+}}{W}+O\left(W^{-2}\right)-z\right| \\
& \geq|| z|-| 1-\frac{(2 k+1) \alpha_{+}}{W}+O\left(W^{-2}\right)|| \geq \frac{(2 k-1 / 2-\varepsilon) \alpha_{1}}{W}
\end{aligned}
$$

with some small fixed $\varepsilon>0$. Thus, by (3.20)

$$
\left|\left(R D^{-1}\right)_{k k+2}\right| \leq \frac{\left|\alpha_{2}\right| \sqrt{(k+1)(k+2)}}{\alpha_{1}(2 k+7 / 2-\varepsilon)}+O\left(k W^{-1}\right)<C<1,
$$

since $\alpha_{2}<\alpha_{1}$ in view of (3.14) and the fact that $\arg c_{ \pm} \in(-\pi / 2, \pi / 2)$. Besides,

$$
\left(R D^{-1}\right)^{m}=0
$$

and we get (3.33) from

$$
\left(\widehat{A}_{+, *}^{(m)}-z\right)^{-1}=\sum_{l=0}^{m} D^{-1}(z)\left(R D^{-1}(z)\right)^{l} .
$$

Thus, we obtain the first bound of $(3.28)$ in view of (3.32) and (3.36). The second bound of (3.28) can be obtained by the same way. The only difference is that, if we defined $R_{-}$and $D_{-}$similarly to (3.34) , then to prove $\left\|D_{-}(z)\right\| \leq C W$ and $\left|\left(R_{-}\left(D_{-}(z)\right)^{-1}\right)_{02}\right| \leq C<1$ we have to use also that $\left|z-\lambda_{0,-}\right| \geq C / W$.

Remark 3.1 Applying the Taylor expansions up to the $m$-th order to the functions $\mathcal{F}(x)$ and $\mathcal{F}(y)$ one can prove that

$$
\left|\left(A_{ \pm}^{(m)}\right)_{j k}\right| \leq(C m / W)^{|j-k| / 2} .
$$

Indeed, it is well known that the Hermite polynomials $\left\{\psi_{k}(x)\right\}_{k=0}^{\infty}$ satisfy the recursion relation

$$
x \psi_{k}(x)=\sqrt{\frac{k+1}{4 \alpha_{1} W}} \psi_{k+1}(x)+\sqrt{\frac{k}{4 \alpha_{1} W}} \psi_{k-1}(x) .
$$

Hence, the operator $\widehat{L}$ of multiplication by $x-a_{+}$has a three diagonal form in the basis $\left\{\psi_{k}^{+}\right\}$, and $\widehat{L}^{l}$ has $2 l+1$ non empty diagonals. The recursion relations combined with (3.20) yield (3.37).

Bound (3.37) implies, in particular, that if $a_{0}$ and $a_{0}^{*}$ are the parts of $a$ and $a^{*}$ which belong to $A^{(11)}$, then

$$
\left\|a-a_{0}\right\| \leq(C m / W)^{m / 2}, \quad\left\|a^{*}-a_{0}^{*}\right\| \leq(C m / W)^{m / 2} .
$$

Proof of Lemma 3.3. According (3.23), to prove Lemma 3.3 we have to prove

$$
\begin{aligned}
& \left\|P_{+} A\left(I_{+}-P_{+}\right)\right\| \leq C W^{-1}, \quad\left\|P_{-} A\left(I_{-}-P_{-}\right)\right\| \leq C W^{-1}, \\
& \left\|\left(I_{+}-P_{+}\right) A P_{+}\right\| \leq C W^{-3 / 2}, \quad\left\|\left(I_{-}-P_{-}\right) A P_{-}\right\| \leq C W^{-3 / 2} .
\end{aligned}
$$


Let us prove the first inequality in (3.39) (the second is similar). Use the bound valid for any $(m+1) \times \infty$ matrix:

$$
\|M\|^{2} \leq \sum_{j=0}^{m}\left\|M_{j}\right\|^{2}
$$

where $M_{j}=M^{*} \psi_{j, \delta}^{+}$. The Parseval identity implies

$$
\left\|\left(P_{+} A^{*}\left(I_{+}-P_{+}\right)\right) \psi_{j, \delta}^{+}\right\|^{2}=\left\|A^{*} \psi_{j, \delta}^{+}\right\|^{2}-\left\|\left(A_{+}^{(m)}\right)^{*} \psi_{j, \delta}^{+}\right\|^{2} .
$$

Using the argument of Lemma 3.2, we get for $j \leq m$

$$
\begin{aligned}
& \left\|A^{*} \psi_{j, \delta}^{+}\right\|^{2}=\left\|\left(A_{*}^{+}\right)^{*} \psi_{j}^{+}\right\|^{2}+O\left((m / W)^{3 / 2}\right) ; \\
& \left\|\left(A_{+}^{(m)}\right)^{*} \psi_{j, \delta}^{+}\right\|^{2}=\left\|\left(A_{+, *}^{(m)}\right)^{*} \psi_{j}^{+}\right\|^{2}+O\left((m / W)^{3 / 2}\right) .
\end{aligned}
$$

Hence, the Parseval identity and the bounds (3.19) - (3.20) yield

$$
\left\|P_{+} A\left(I_{+}-P_{+}\right)\right\| \leq\left(\sum_{j=0}^{m} \sum_{k>m}\left|A_{* j k}^{+}\right|^{2}\right)^{1 / 2} \leq C m / W+O\left(m(m / W)^{3 / 2}\right),
$$

which gives (3.39) (recall that $m$ is $W$-independent). The bounds (3.40) can be obtained similarly, if we use the fact that $A_{* j k}^{+}=0$ for $j>k$.

Proof of Lemma 3.4. Consider any $\|u\|=1$ such that $\left(u, \psi_{k, \delta}^{+}\right)=\left(u, \psi_{k, \delta}^{-}\right)=0$ for $k=0,1, \ldots, m$. Split $x \in \mathbb{R}$ into three sub domains, according to the value of the function $\mathcal{F}(x)$ :

$$
\begin{aligned}
& \Lambda_{1}=\{x:|\mathcal{F}(x)| \geq 1-\delta / 2\} ; \\
& \Lambda_{2}=\{x: 1-\delta \leq|\mathcal{F}(x)|<1-\delta / 2\} ; \\
& \Lambda_{3}=\{x:|\mathcal{F}(x)|<1-\delta\},
\end{aligned}
$$

and let $u_{s}$ be the projections of $u$ corresponding to $\Lambda_{s}, s=1,2,3$.

By (3.5)

$$
\begin{aligned}
\|A u\|^{2} \leq\left\|\mathcal{F}^{2} u\right\|^{2} & \leq\left\|u_{1}\right\|^{2}+(1-\delta / 2)^{2}\left\|u_{2}+u_{3}\right\|^{2} \\
& =1-\left(1-(1-\delta / 2)^{2}\right)\left\|u_{2}+u_{3}\right\|^{2} \\
& \Rightarrow\left\|u_{2}+u_{3}\right\|^{2} \leq C_{0}\left(1-\|A u\|^{2}\right) .
\end{aligned}
$$

Set

$$
u_{0}:=u_{1}+u_{2}, \quad u_{0}^{+}=u_{0} 1_{\omega_{\delta}^{+}}, \quad u_{0}^{-}=u_{0} 1_{\omega_{\delta}^{-}},
$$

Note that according to the choice of $u$

$$
\left(u_{0}^{+}, \psi_{k, \delta}^{+}\right)=O\left(e^{-c W}\right), \quad\left(u_{0}^{-}, \psi_{k, \delta}^{-}\right)=O\left(e^{-c W}\right), \quad k=0, \ldots m .
$$

Lemma 3.5 For any $u_{0}^{+}, u_{0}^{-}$satisfying (3.44) we have

$$
\left\|A^{(22)} u_{0}^{ \pm}\right\|^{2} \leq\left(1-\frac{C m^{1 / 3}}{W}\right)\left\|u_{0}^{ \pm}\right\|^{2} .
$$


The proof of Lemma 3.5 is given after the end of the proof of Lemma 3.4. Now we assume for the moment that Lemma 3.5 is proved and finish the proof of Lemma 3.4.

According to (3.44) the assumptions of Lemma 3.5 are satisfied. Moreover,

$$
\begin{aligned}
\Re\left(A u_{0}, A u_{3}\right) & =\Re\left(A u_{1}, A u_{3}\right)+\Re\left(A u_{2}, A u_{3}\right) \\
& =O\left(e^{-c W^{2}}\right)+\Re\left(A u_{2}, A u_{3}\right) \leq O\left(e^{-c W^{2}}\right)+\frac{1}{2}\left\|u_{2}+u_{3}\right\|^{2},
\end{aligned}
$$

since $\|A\| \leq 1$. Thus we have by (3.42), (3.45)

$$
\begin{aligned}
\|A u\|^{2} & =\left\|A\left(u_{0}^{+}+u_{0}^{-}+u_{3}\right)\right\|^{2} \\
& =\left\|A u_{0}^{+}\right\|^{2}+\left\|A u_{0}^{-}\right\|^{2}+2 \Re\left(A u_{0}, A u_{3}\right)+\left\|A u_{3}\right\|^{2}+O\left(e^{-c W}\right) \\
& \leq\left(1-C m^{1 / 3} / W\right)\left(\left\|u_{0}^{+}\right\|^{2}+\left\|u_{0}^{-}\right\|^{2}\right)+2\left\|u_{2}+u_{3}\right\|^{2}+O\left(e^{-c W}\right) \\
& \leq 1-C m^{1 / 3} / W+2 C_{0}\left(1-\|A u\|^{2}\right) \\
& \Rightarrow\left(1-\|A u\|^{2}\right)\left(1+2 C_{0}\right) \geq C m^{1 / 3} / W \\
& \Rightarrow\|A u\|^{2} \leq 1-C_{1} m^{1 / 3} / W .
\end{aligned}
$$

Here in the third line we used that

$$
\left\|A u_{3}\right\|^{2} \leq\left\|u_{3}\right\|^{2} \leq\left\|u_{2}+u_{3}\right\|^{2} .
$$

Now since, by definition, the block $\mathcal{A}^{(22)}$ corresponds to $u$, which are orthogonal to $\left\{\psi_{k, \delta}^{+}\right\}_{k=0}^{m}$ and $\left\{\psi_{k, \delta}^{-}\right\}_{k=0}^{m}$, the last inequality proves that

$$
\begin{aligned}
& \|A(1-P)\| \leq 1-C_{1} m^{1 / 3} / W \\
& \Rightarrow\left\|A^{(22)}\right\|=\|(1-P) A(1-P)\| \leq 1-C_{1} m^{1 / 3} / W \\
& \Rightarrow\left\|\left(A^{(22)}-z\right)^{-1}\right\| \leq\left|z^{-1}\right| \sum_{s=0}^{\infty}\left(\left\|A^{(22)}\right\| /|z|\right)^{s} \leq C_{1} W / m^{1 / 3},
\end{aligned}
$$

which gives the assertion of Lemma 3.4. We are left to prove Lemma 3.5.

Proof of Lemma 3.5. We prove first the relation for $u_{0}^{+}$. Choose $c_{0}>0$ sufficiently small to provide

$$
\Re f(x) \geq \frac{c_{0}}{2}\left(x-a_{+}\right)^{2}, \quad x \geq 0, \quad \mathcal{F}_{0}=e^{-c_{0}\left(x-a_{+}\right)^{2} / 2}, \quad A_{0}=\mathcal{F}_{0} B \mathcal{F}_{0}
$$

Consider the basis $\left\{\widetilde{\psi}_{k}\right\}_{k \geq 0}$ in which $A_{0}^{*} A_{0}$ is diagonal. The straightforward calculus gives (cf. Lemma 3.1)

$$
\begin{aligned}
& \widetilde{\psi}_{k}\left(x-a_{+}\right)=\widetilde{h}_{k}^{-1 / 2} e^{\widetilde{\alpha} W\left(x-a_{+}\right)^{2}}\left(\frac{d}{d x}\right)^{k} e^{-2 \widetilde{\alpha} W\left(x-a_{+}\right)^{2}} \\
& \widetilde{\alpha}=\sqrt{\frac{c_{0}}{2}}\left(1+\frac{c_{0}}{2 W^{2}}\right)^{1 / 2}, \quad \widetilde{h}_{k}=k !(4 \widetilde{\alpha} W)^{k-1 / 2} \sqrt{2 \pi},
\end{aligned}
$$

Now, by the assumptions of the lemma $\left(u_{0}^{+}, \psi_{k}^{+}\right)=\left(u_{0}^{+}, \psi_{k, \delta}^{+}\right)+O\left(e^{-c W}\right)=O\left(e^{-c W}\right)$ for $k=0,1, \ldots, m$, thus

$$
u_{0}^{+}=\left(1-P_{m}\right) u_{0}^{+}+O\left(e^{-c W}\right),
$$


Hence, setting $l=\left[m^{1 / 3} / \tilde{C} \sqrt[3]{2}\right]$ with $\tilde{C}$, depending only on $C$ in (3.21), and denoting by $P_{l}$ the orthogonal projection on the linear span of $\left\{\widetilde{\psi}_{k}\right\}_{k=0}^{l}$, we get by (3.21)

$$
\left\|P_{l} u_{0}^{+}\right\|^{2} \leq \frac{\left\|u_{0}^{+}\right\|^{2}}{2}
$$

Moreover, the commutators $[\mathcal{F}, B]$ and $\left[\mathcal{F}_{0}, B\right]$ admit the bounds

$$
\begin{aligned}
\|[\mathcal{F}, B]\| & \leq \sup _{x} W \int|\mathcal{F}(x)-\mathcal{F}(y)| e^{-W^{2}(x-y)^{2}} d y \\
& \leq C W \int|x-y| e^{-W^{2}(x-y)^{2}} d y \leq C_{*} / W
\end{aligned}
$$

and similarly

$$
\left\|\left[\mathcal{F}_{0}, B\right]\right\| \leq C_{*} / W
$$

Thus

$$
\begin{aligned}
\left\|A u_{0}^{+}\right\|^{2} & =\left(\mathcal{F}^{*} B \mathcal{F}^{*} \mathcal{F} B \mathcal{F} u_{0}^{+}, u_{0}^{+}\right) \leq\left(B \mathcal{F}^{*} \mathcal{F}^{*} \mathcal{F} \mathcal{F} B u_{0}^{+}, u_{0}^{+}\right)+2 C_{*} W^{-1}\left\|u_{0}^{+}\right\|^{2} \\
& \leq\left(B F_{0}^{4} B u_{0}^{+}, u_{0}^{+}\right)+2 C_{*} W^{-1}\left\|u_{0}^{+}\right\|^{2} \leq\left(\mathcal{F}_{0} B \mathcal{F}_{0}^{2} B \mathcal{F}_{0} u_{0}^{+}, u_{0}^{+}\right)+4 C_{*} W^{-1}\left\|u_{0}^{+}\right\|^{2} \\
& =\left(A_{0} u_{0}^{+}, A_{0} u_{0}^{+}\right)+4 C_{*} W^{-1}\left\|u_{0}^{+}\right\|^{2}=\left\|A_{0} u_{0}^{+}\right\|^{2}+4 C_{*} W^{-1}\left\|u_{0}^{+}\right\|^{2} .
\end{aligned}
$$

Then, since $\left[A_{0}, P_{l}\right]=0,\left\|A_{0}\left(1-P_{l}\right)\right\| \leq 1-l \sqrt{2 c_{0}} / 2 W\left(\right.$ see $(\underline{3.20})$ ), and $\left\|P_{l} u_{0}^{+}\right\| \leq \frac{1}{2}\left\|u_{0}^{+}\right\|^{2}$, we have

$$
\begin{aligned}
\left\|A u_{0}^{+}\right\|^{2} & =\left\|A_{+}\left(P_{l} u_{0}^{+}+\left(1-P_{l}\right) u_{0}^{+}\right)\right\|^{2}+O\left(e^{-c W}\right) \\
& \leq\left\|A_{0}\left(P_{l} u_{0}^{+}+\left(1-P_{l}\right) u_{0}^{+}\right)\right\|^{2}+4 C_{*} W^{-1}\left\|u_{0}^{+}\right\|^{2} \\
& =\left\|A_{0} P_{l} u_{0}^{+}\right\|^{2}+\left\|A_{0}\left(1-P_{l}\right) u_{0}^{+}\right\|^{2}+4 C_{*} W^{-1}\left\|u_{0}^{+}\right\|^{2} \\
& \leq\left\|P_{l} u_{0}^{+}\right\|^{2}+\left(1-l \sqrt{2 c_{0}} / 2 W\right)\left(\left\|u_{0}^{+}\right\|^{2}-\left\|P_{l} u_{0}^{+}\right\|^{2}\right)+4 C_{*} W^{-1}\left\|u_{0}^{+}\right\|^{2} \\
& \leq\left(1-l \sqrt{2 c_{0}} / 2 W\right)\left\|u_{0}^{+}\right\|^{2}+\left\|P_{l} u_{0}^{+}\right\|^{2} l \sqrt{2 c_{0}} / 2 W+4 C_{*} W^{-1}\left\|u_{0}^{+}\right\|^{2} \\
& \leq\left(1-l \sqrt{2 c_{0}} / 4 W+2 C_{*} W^{-1}\right)\left\|u_{0}^{+}\right\|^{2} \leq\left(1-l \sqrt{2 c_{0}} / 5 W\right)\left\|u_{0}^{+}\right\|^{2}
\end{aligned}
$$

if $l$ is sufficiently large.

\subsection{Analysis of $K_{*}$}

Proposition 3.2 If we consider $K_{*}\left(t, U_{1}, U_{2}\right)$ of 3.10 as a kernel of the self-adjoint integral operator in $L_{2}[\stackrel{\circ}{U}(2), \mu(U)]$, its eigenvectors $\left\{\phi_{\bar{j}}(U)\right\}(\bar{j}=(j, k), j=0,1, \ldots, k=-j, \ldots, j)$ do not depend on $a_{1}, a_{2}, b_{1}, b_{2}$ and the corresponding eigenvalues $\left\{\lambda_{\bar{j}}(t)\right\}$ have the form

$$
\lambda_{\overline{0}}(t)=1-e^{-W^{2} t},
$$

and for $t>d>0$ we have

$$
\begin{aligned}
& \lambda_{\bar{j}}(t)=\left(1-e^{-W^{2} t}\right)\left(1-\frac{j(j+1)}{W^{2} t}\left(1+O\left(j^{2} / W^{2} t\right)\right)\right. \\
& \left(\nu, \phi_{0}\right)=0 .
\end{aligned}
$$


The proof of the proposition is given in Appendix.

It follows from Proposition 3.2 that, if we introduce the basis in $L_{2}\left[\mathbb{R}^{2}, p\right] \times L_{2}[\stackrel{\circ}{U}(2), d U]$

$$
\begin{aligned}
& \Psi_{\bar{k}, \bar{j}}(a, b, U)=\Psi_{\bar{k}}(a, b) \phi_{\bar{j}}(U), \\
& \Psi_{\bar{k}}(a, b)=\sqrt{\frac{2}{\pi}}(a-b)^{-1} \psi_{k_{1}}(a) \psi_{k_{2}}(b),
\end{aligned}
$$

where $\left\{\psi_{k}(x)\right\}_{k=0}^{\infty}$ is some basis in $L_{2}[\mathbb{R}]$, then the matrix of $K$ of (3.10) in this basis has a "block diagonal structure", which means that

$$
\begin{aligned}
& \left(K \Psi_{\bar{k}^{\prime}, \bar{j}}, \Psi_{\bar{k}, \bar{j}_{1}}\right)_{p}=0, \quad j \neq j_{1} \\
& \left(K \Psi_{\bar{k}^{\prime} \bar{j}}, \Psi_{\bar{k}, \bar{j}}\right)_{p}=\left(K_{j} \Psi_{\bar{k}^{\prime}}, \Psi_{\bar{k}}\right)_{p} \\
= & \int \lambda_{\bar{j}}(t) A\left(a_{1}, a_{2}\right) A\left(b_{1}, b_{2}\right) \psi_{k_{1}}\left(a_{1}\right) \psi_{k_{2}}\left(b_{1}\right) \psi_{k_{1}^{\prime}}\left(a_{2}\right) \psi_{k_{2}^{\prime}}\left(b_{2}\right) d a_{1} d b_{1} d a_{2} d b_{2} .
\end{aligned}
$$

\section{Analysis of $K$}

Theorem 4.1 For the operators $K, K(\xi)$ of (2.3), 2.4) there is an absolute $\varepsilon>0$ such that

$$
\begin{aligned}
& \lambda_{0}(K)=\lambda_{0,+} \lambda_{0,-}+O\left(e^{-c \log ^{3} W}\right), \quad\left|\lambda_{1}(K)\right| \leq\left|\lambda_{0}(K)\right|-\varepsilon / W^{2}, \\
& \left|\lambda_{0}(K)-\lambda_{0}(K(\xi))\right| \leq C\left((W / n)^{2}+1 / n \sqrt{W}\right), \\
& \left|\lambda_{1}(K(\xi))\right| \leq\left|\lambda_{0,+} \lambda_{0,-}\right|-\varepsilon / 2 W^{2},
\end{aligned}
$$

where $\lambda_{0, \pm}$ are defined in (3.13).

In particular, Theorem 4.1 gives (2.6), (2.8), hence the assertion of Theorem 1.2 .

\subsection{Proof of Theorem 4.1}

Choose $W, n$-independent $\delta>0$, which is small enough to provide that the domain

$$
\Omega_{\delta}=\{X:|\mathcal{F}(X)|>1-\delta\}
$$

contains three non intersecting sub domains $\Omega_{\delta}^{ \pm}, \Omega_{\delta}^{+}, \Omega_{\delta}^{-}$, such that each of $\Omega_{\delta}^{+}, \Omega_{\delta}^{-}$contains one of the points $X_{+}=a_{+} I$ and $X_{-}=a_{-} I$ of maximum $\mathcal{F}(X)$, and $\Omega_{\delta}^{ \pm}$contains the "surface" $X^{*}(U)=U D U^{*}$ with $D=\operatorname{diag}\left\{a_{+}, a_{-}\right\}$, and $U \in \stackrel{\odot}{U}(2)$ (see (3.1)). Set also

$$
m=\left[\log ^{2} W\right] .
$$

Consider a system of functions

$$
\begin{aligned}
& \left\{\Psi_{\bar{k}, \bar{j}, \delta}\right\}_{|\bar{k}| \leq m, j \leq(m W)^{1 / 2}}, \\
& \bar{k}=\left(k_{1}, k_{2}\right),|\bar{k}|=\max \left\{k_{1}, k_{2}\right\}, \quad \bar{j}=(j, l), l=-j, \ldots, j,
\end{aligned}
$$

obtained by the Gramm-Schmidt procedure from

$$
\left\{1_{\Omega_{\delta}^{ \pm}} \Psi_{\bar{k}, \bar{j}}\right\}_{|\bar{k}| \leq m ; j \leq(m W)^{1 / 2},}
$$


where

$$
\begin{aligned}
& \Psi_{\bar{k}, \bar{j}}(a, b, U)=\Psi_{\bar{k}}(a, b) \phi_{\bar{j}}(U), \\
& \Psi_{\bar{k}}(a, b)=\sqrt{\frac{2}{\pi}}(a-b)^{-1} \psi_{k_{1}}^{+}\left(a-a_{+}\right) \psi_{k_{2}}^{-}\left(b-a_{-}\right) .
\end{aligned}
$$

Similarly, consider the system of functions $\left\{\Psi_{\bar{k}, \delta}^{+}\right\}_{|\bar{k}| \leq m}$ (with $\bar{k}=\left(k_{1}, k_{2}, k_{3}, k_{4}\right),|\bar{k}|=$ $\left.\max \left\{k_{i}\right\}\right)$ obtained by the Gramm-Schmidt procedure from

$$
\left\{1_{\Omega_{\delta}^{+}} \psi_{k_{1}}^{+}\left(a-a_{+}\right) \psi_{k_{2}}^{+}\left(b-a_{+}\right) \psi_{k_{3}}^{+}(x) \psi_{k_{4}}^{+}(y)\right\}_{|\bar{k}| \leq m}
$$

and define $\left\{\Psi_{\bar{k}, \delta}^{-}\right\}_{|\bar{k}| \leq m}$ by the same way. Denote $P_{ \pm}, P_{+}$, and $P_{-}$the projections on the subspaces, spanned on these three systems. Evidently these three projection operators are orthogonal to each other. Set

$$
P=P_{ \pm}+P_{+}+P_{-}, \quad \mathcal{L}_{1}=P \mathcal{H}, \quad \mathcal{L}_{2}=(1-P) \mathcal{H}, \quad \mathcal{H}=\mathcal{L}_{1} \oplus \mathcal{L}_{2},
$$

where $\mathcal{H}$ is defined in (2.1).

Besides, note that for any $f$, supported in some domain $\Omega$, and any $C>0$ (cf. (3.29))

$$
(K f)(X)=O\left(e^{-c W^{2}}\right) \text { for } X: \operatorname{dist}\{X, \Omega\} \geq C>0 .
$$

Now consider the operator $K$ as a block operator with respect to the decomposition (4.4). It has the form

$$
\begin{aligned}
& K^{(11)}=K_{ \pm}+K_{+}+K_{-}+O\left(e^{-c W}\right) \\
& K_{ \pm}:=P_{ \pm} K P_{ \pm}, \quad K_{+}=P_{+} K P_{+}, \quad K_{-}:=P_{-} K P_{-} \\
& K^{(12)}=P_{ \pm} K\left(I_{ \pm}-P_{ \pm}\right)+P_{+} K\left(I_{+}-P_{+}\right)+P_{-} K\left(I_{-}-P_{-}\right)+O\left(e^{-c W}\right) \\
& K^{(21)}=\left(I_{ \pm}-P_{ \pm}\right) K P_{ \pm}+\left(I_{+}-P_{+}\right) K P_{+}+\left(I_{-}-P_{-}\right) K P_{-}+O\left(e^{-c W}\right)
\end{aligned}
$$

where $I_{ \pm}, I_{+}$, and $I_{-}$are operators of multiplication by $1_{\Omega_{\delta}^{ \pm}}, 1_{\Omega_{\delta}^{+}}$, and $1_{\Omega_{\delta}^{-}}$respectively. Indeed, it is easy to see from (4.5) and from the relation

$$
\psi_{k}(x)=O\left(e^{-c W}\right) \text { for }|x| \geq C>0, \quad k \leq m
$$

that, e.g. , $P_{+} K P_{-} f=O\left(e^{-c W}\right), P_{ \pm} K\left(I_{+}-P_{+}\right) f=O\left(e^{-c W}\right)$, etc (cf. (3.23) $)$.

Moreover, by (3.53) the block $K_{ \pm}$also has a block diagonal structure:

$$
K_{ \pm}=\sum_{j=0}^{(m W)^{1 / 2}} K_{ \pm}^{(j)}, \quad K_{ \pm}^{(j)}=\mathcal{P}_{j} P_{ \pm} K P_{ \pm} \mathcal{P}_{j}
$$

Here and below we denote by $\mathcal{P}_{j}$ the projection on linear span of $\left\{\Psi(a, b) \phi_{(j, l)}(U)\right\}_{l=-j}^{j}$.

Take some $W, n$-independent sufficiently small $\varepsilon$, and consider $z$, satisfying the conditions

$$
z \in \mathcal{D}_{\varepsilon}:=\left\{z:\left|\lambda_{0,+} \lambda_{0,-}\right|-\varepsilon W^{-2} \leq|z| \leq 1 \wedge\left|z-\lambda_{0,+} \lambda_{0,-}\right|>\varepsilon W^{-2}\right\}
$$

Following Proposition 3.1, introduce also the vectors

$$
\kappa=K \Psi_{\overline{0}, 0, \delta}-\left(K \Psi_{\overline{0}, 0, \delta}, \Psi_{\overline{0}, 0, \delta}\right) \Psi_{\overline{0}, 0, \delta}, \quad \kappa^{*}=K^{\dagger} \Psi_{\overline{0}, 0, \delta}-\left(K^{\dagger} \Psi_{\overline{0}, 0, \delta}, \Psi_{\overline{0}, 0, \delta}\right) \Psi_{\overline{0}, 0, \delta} .
$$

The proof of Theorem 4.1 is based on the following three lemmas. 
Lemma 4.1 Given $z \in \mathcal{D}_{\varepsilon}$ consider $G^{(j)}(z)=\left(K_{ \pm}^{(j)}-z\right)^{-1}$. Then each $G^{(j)}$ can be represented in the form (3.16), and the corresponding matrices $\widehat{G}_{j}$ and functions $F_{j}$ of (3.15) satisfy the bound

$$
\begin{aligned}
& \left\|\widehat{G}^{(j)}\right\| \leq C m W, \quad\left|\widehat{G}_{\bar{k} \bar{k}^{\prime}}^{(j)}\right| \leq C m W q^{\left|\bar{k}-\bar{k}^{\prime}\right| / 2}, \quad 0<q<1, \\
& \left|F_{j}(z)\right|>C W^{-2}, \quad\left\|G^{(j)}\right\| \leq C W^{2} .
\end{aligned}
$$

In addition,

$$
\left\|\left(K_{+}-z\right)^{-1}\right\| \leq C W, \quad\left\|\left(K_{-}-z\right)^{-1}\right\| \leq C W .
$$

Lemma 4.2 Fot the off-diagonal blocks of the operator $K$ (see (4.6)) we have

$$
\begin{aligned}
& \left\|K^{(21)}\right\| \leq C m^{3 / 2} / W^{3 / 2}, \quad\left\|K^{(12)}\right\| \leq C m / W, \\
& \|\kappa\| \leq C W^{-3 / 2}, \quad\left\|\kappa^{*}\right\| \leq C W^{-1} .
\end{aligned}
$$

Moreover, there is some absolute $p>0$ such that

$$
\left\|\left(\widehat{K}^{(11)}-z\right)^{-1} \widehat{K}^{(12)}\right\| \leq C m^{p} .
$$

\section{Lemma 4.3}

$$
\left\|K^{(22)}\right\| \leq 1-C m^{1 / 3} / W
$$

Defer the proofs of the lemmas to the next section and show how one can finish the proof of Theorem 4.1, using the lemmas.

It is easy to see that the first line of (4.12) and (4.14) yield

$$
\left\|\left(K^{(22)}-z\right)^{-1} K^{(21)}\right\| \leq C m^{p} W^{-1 / 2} .
$$

Here and below we denote by $p$ some absolute exponents which could be different in different formulas. The bound and (4.13) imply that $\widehat{K}-z$ can be represented in the form

$$
\widehat{K}-z=\left(\begin{array}{cc}
\widehat{K}^{(11)}-z & 0 \\
0 & K^{(22)}-z
\end{array}\right)\left(\begin{array}{cc}
I & O\left(m^{p}\right) \\
O\left(m^{p} W^{-1 / 2}\right) & I
\end{array}\right) .
$$

Both matrices here are invertible, and the inverse of the second one has a similar form. Hence

$$
\widehat{G}(z)=(\widehat{K}-z)^{-1}=\left(\begin{array}{cc}
I \cdot(1+o(1)) & O\left(m^{p}\right) \\
O\left(m^{p} W^{-1 / 2}\right) & I \cdot(1+o(1))
\end{array}\right)\left(\begin{array}{cc}
\left(\widehat{K}^{(11)}-z\right)^{-1} & 0 \\
0 & \left(K^{(22)}-z\right)^{-1}
\end{array}\right) .
$$

Thus we get from the representation and (4.10)

$$
\|\widehat{G}\| \leq C W^{2} .
$$

Moreover, if we set $\widetilde{G}=\left(\widehat{K}+\widetilde{K}^{0}-z\right)^{-1}$, where $\widetilde{K}^{0}$ is $\widetilde{K}$ without the "line" and the "column", corresponding to $\Psi_{\overline{0}, 0, \delta}$, then taking into account that $\left\|\widetilde{K}^{0}\right\| \leq C / n \ll W^{-2}$, we obtain

$$
\widetilde{G}=\widehat{G}\left(1+\widetilde{K}^{0} \widehat{G}\right)^{-1}=\widehat{G}\left(1+H_{0} \widehat{G}\right), \quad\left\|H_{0}\right\| \leq n^{-1},
$$


and so for $W^{2} \ll n$

$$
\|\widetilde{G}\| \leq C W^{2} .
$$

Notice that the definition of $\Psi_{\overline{0}, 0, \delta}$ (see (4.2), (4.3)), and $\mathcal{P}_{j}$ (see (4.7)) and (4.9), combined with (3.38) yield

$$
\begin{aligned}
\kappa & =\mathcal{P}_{0} \kappa, \quad \kappa^{*}=\mathcal{P}_{0} \kappa^{*}, \\
\Rightarrow \hat{G} \kappa & =\hat{G}^{(0)} \kappa+O\left((C W)^{-m}\right), \quad \hat{G} \kappa^{*}=\hat{G}^{(0)} \kappa^{*}+O\left(e^{-c \log ^{3} W}\right) .
\end{aligned}
$$

Consider the function $F_{K}(z)$ of the form (3.15), constructed for $K$. Then (3.53) and (3.51) yield

$$
\begin{aligned}
F_{K}(z) & :=\left(K \Psi_{\overline{0}, 0, \delta}, \Psi_{\overline{0}, 0, \delta}\right)-\left((\widehat{K}-z)^{-1} \kappa, \kappa^{*}\right) \\
& =\left(A_{+}^{(m)}\right)_{00}\left(A_{-}^{(m)}\right)_{00}-z-\left(\widehat{G}^{(0)} \kappa_{0}, \kappa_{0}^{*}\right)+O\left(e^{-c \log ^{3} W}\right) \\
& =: F_{ \pm}(z)+O\left(e^{-c \log ^{3} W}\right)
\end{aligned}
$$

where $A_{+}^{(m)}$ and $A_{-}^{(m)}$ are defined in (3.23). But since $F_{ \pm}$is constructed according to Proposition 3.1 for $A_{+}^{(m)} \otimes A_{-}^{(m)}$, we know that $F_{ \pm}(z)$ has no roots in $D_{\varepsilon}$ and has exactly one root in $\omega_{\varepsilon}=\left\{z:\left|z-\lambda_{0,+} \lambda_{0,-}\right| \leq \varepsilon W^{-2}\right\}$. In addition

$$
\begin{aligned}
& \frac{d}{d z} F_{ \pm}(z)=-1+\left(\left(\widehat{G}^{(0)}\right)^{2} \kappa_{0}, \kappa_{0}^{*}\right)=-1+O\left(W^{-1 / 2}\right) \\
& \Rightarrow\left|F_{ \pm}(z)\right|>\varepsilon W^{-2} / 2, z \in \mathcal{D}_{\varepsilon}
\end{aligned}
$$

Hence, by the Rouchet theorem, we conclude that $F_{K}(z)$ has no roots in $\mathcal{D}_{\varepsilon}$ and has exactly one root in $\omega_{\varepsilon}$. This gives us the first line of (4.1).

To apply Proposition 3.1 to $K+\widetilde{K}$, denote by $\tilde{\kappa}$ and $\tilde{\kappa}^{*}$ the vectors

$$
\tilde{\kappa}=\widetilde{K} \Psi_{\overline{0}, 0, \delta}-\left(\widetilde{K} \Psi_{\overline{0}, 0, \delta}, \Psi_{\overline{0}, 0, \delta}\right) \Psi_{\overline{0}, 0, \delta}, \quad \tilde{\kappa}^{*}=\widetilde{K}^{*} \Psi_{\overline{0}, 0, \delta}-\left(\widetilde{K}^{*} \Psi_{\overline{0}, 0, \delta}, \Psi_{\overline{0}, 0, \delta}\right) \Psi_{\overline{0}, 0, \delta}
$$

Then by the second line of (3.52)

$$
\mathcal{P}_{0} \tilde{\kappa}=0, \quad \mathcal{P}_{0} \tilde{\kappa}^{*}=0 .
$$

Moreover, we have

$$
\|\tilde{\kappa}\|=O\left(n^{-1}\right), \quad\left\|\tilde{\kappa}^{*}\right\|=O\left(n^{-1}\right) .
$$

By Proposition 3.1, one should study zeros of the function

$$
\widetilde{F}_{K}(z)=\left((K+\widetilde{K}) \Psi_{\overline{0}, 0}, \Psi_{\overline{0}, 0}\right)-z-\left(\widetilde{G}(z)(\kappa+\tilde{\kappa}),\left(\kappa^{*}+\tilde{\kappa}^{*}\right)\right) .
$$

Let us prove that there is $C>0$ such that

$$
\left|F_{K}(z)-\widetilde{F}_{K}(z)\right| \leq C\left((W / n)^{2}+1 / n \sqrt{W}+O\left(e^{-c \log ^{3} W}\right)\right) .
$$

By (4.17) and (4.22) we have,

$$
\left|\left(\widetilde{G} \tilde{\kappa}, \tilde{\kappa}^{*}\right)\right| \leq\|\widetilde{G}\| / n^{2} \leq C W^{2} / n^{2} .
$$


Moreover, the representation (4.16) and relations (4.18), (4.21), and (4.10) yield

$$
\begin{aligned}
\left|\left(\widetilde{G} \kappa, \tilde{\kappa}^{*}\right)\right| & \left.\leq\left|\left(\widehat{G} \kappa, \tilde{\kappa}^{*}\right)\right|+\mid\left(\widehat{G} H_{0} \widehat{G} \kappa, \tilde{\kappa}^{*}\right)\right) \mid \\
& \leq\left|\left(\widehat{G}^{(0)} \kappa, \tilde{\kappa}^{*}\right)\right|+\left|\left(\widehat{G} H_{0} \widehat{G}^{(0)} \kappa, \tilde{\kappa}^{*}\right)\right|+O\left(e^{-c \log ^{3} W} / n\right) \\
& \leq\|\widehat{G}\| \cdot\left\|H_{0}\right\| \cdot\left\|\widehat{G}^{(0)}\right\| \cdot\|\kappa\| \cdot \| \tilde{\kappa}^{*}||+O\left(e^{-c \log ^{3} W} / n\right) \\
& \leq C_{2} W^{3 / 2} / n^{2}+O\left(e^{-c \log ^{3} W} / n\right) .
\end{aligned}
$$

Here we have used that

$$
\widehat{G}^{(0)} \kappa=\mathcal{P}_{0} \widehat{G}^{(0)} \kappa \Rightarrow\left(\widehat{G}^{(0)} \kappa, \tilde{\kappa}^{*}\right)=0 .
$$

Similarly to (4.24) we get

$$
\begin{aligned}
& \left|\left(\widetilde{G} \tilde{\kappa}, \kappa^{*}\right)\right| \leq C_{3}(W / n)^{2}+O\left(e^{-c \log ^{3} W} / n\right), \\
& \left|\left((\widetilde{G}-\widehat{G}) \kappa, \kappa^{*}\right)\right| \leq C_{4} / n \sqrt{W} .
\end{aligned}
$$

Besides, by (3.52),

$$
\left(\widetilde{K} \Psi_{\overline{0}, 0}, \Psi_{\overline{0}, 0}\right)=O\left(n^{-2}\right)
$$

Thus, (4.23) is proved.

Let $z_{0}$ be a root of $F_{K}(z)$. Then (4.19) and (4.20) gives us for any $z:\left|z-z_{0}\right|=$ $2 C\left((W / n)^{2}+1 / n \sqrt{W}\right)$ :

$$
\left|F_{K}(z)\right| \geq 2 C\left((W / n)^{2}+1 / n \sqrt{W}\right)\left(1-O\left(W^{-1 / 2}\right)\right)>\left|\widetilde{F}_{K}(z)-F_{K}(z)\right|
$$

Then, by the Rouchet theorem, $\widetilde{F}_{K}(z)$ has exactly one root in the circle

$$
\left.\left|z-z_{0}\right| \leq 2 C\left((W / n)^{2}+1 / n \sqrt{W}\right)\right)
$$

and has no roots in $\mathcal{D}_{\varepsilon}$, defined in (4.8).

\subsection{Proofs of Lemmas 4.14 .3}

Proof of Lemma 4.1. Let us first prove (4.10). To this end we use representation of $K$ in polar coordinates (see (3.9) - (3.10) ). According to (3.52)

$$
\lambda_{\bar{j}}(t)=\lambda_{j}^{*}+O\left(j^{2}\left(t-\left(a_{+}-a_{-}\right)^{2}\right) / W^{2}\right),
$$

where $t$ is defined in (3.11), and

$$
\lambda_{j}^{*}=1-\frac{j(j+1)}{W^{2}\left(a_{+}-a_{-}\right)^{2}}
$$

Definition 4.1 We will denote by $O_{*}\left((m / W)^{3 / 2}\right)$ any operator $T$ satisfying the following property: there exist $p_{1}, p_{2}>0$ such that

$$
\left\|T P_{ \pm}\right\| \leq C m^{p_{1}} W^{-3 / 2}, \quad\left|\left(T P_{ \pm}\right)_{\bar{k}, \bar{k}^{\prime}}\right| \leq m^{p_{2}}(C m / W)^{\left|\bar{k}-\bar{k}^{\prime}\right|}
$$


Using (3.53) and the fact that, according to Remark 3.1, the operator $\tilde{T}$ of multiplication by $\left(t-\left(a_{+}-a_{-}\right)^{2}\right)$ is $O_{*}\left((m / W)^{3 / 2}\right)$, we obtain that

$$
K_{\bar{k}, \bar{k}^{\prime}}^{(j)}=\lambda_{j}^{*} \cdot\left(K_{\bar{k}, \bar{k}^{\prime}}^{(0)}+O_{*}\left((m / W)^{3 / 2}\right)=\lambda_{j}^{*} \cdot\left(A_{+*}^{(m)} \otimes A_{-*}^{(m)}+O_{*}\left((m / W)^{3 / 2}\right) .\right.\right.
$$

Hence

$$
\widehat{G}^{(j)}(z)=\left(\lambda_{j}^{*}\right)^{-1} \cdot\left(A_{+*}^{(m) \otimes A_{-*}^{(m)}}+O_{*}\left((m / W)^{3 / 2}\right)-z_{j}\right)^{-1}, \quad z_{j}=z / \lambda_{j}^{*},
$$

where $A_{+*}^{\left(\widetilde{m)} \otimes A_{-*}^{(m)}\right.}$ means the matrix $A_{+*}^{(m)} \otimes A_{-*}^{(m)}$ without the line and the column, corresponding to $\psi_{0}^{+} \otimes \psi_{0}^{-}$.

Proposition 4.1 Take $\mathcal{A}=A_{\sigma_{1} *}^{(m)} \otimes A_{\sigma_{2} *}^{(m)} \otimes \cdots \otimes A_{\sigma_{s} *}^{(m)}$ with $\sigma_{i}=+,-, i=1, \ldots, s$ and $s$ independent of $n, W, m$. Let

$$
\left|z-\lambda_{0}(\mathcal{A})\right| \leq c_{0} / W^{2} \vee|z|>\left|\lambda_{0}(\mathcal{A})\right|+C_{0} / W
$$

$\widehat{\mathcal{A}}$ be $\mathcal{A}$ without the line and the column, corresponding to $\psi_{0}^{\sigma_{1}} \otimes \cdots \otimes \psi_{0}^{\sigma_{s}}$, and $\widehat{\mathcal{G}}(z)=$ $(\widehat{\mathcal{A}}-z)^{-1}, F(z)$ be defined as in (3.15).

Then there is $0<q<1$ such that

$$
\begin{aligned}
& \|\widehat{\mathcal{G}}(z)\| \leq C m W, \quad\left|(\widehat{\mathcal{G}}(z))_{\bar{k}, \bar{k}^{\prime}}\right| \leq W m^{s} q^{\left|\bar{k}-\bar{k}^{\prime}\right| / 2}, \\
& |F(z)| \geq C\left|z-\lambda_{0}(\mathcal{A})\right|^{-1} .
\end{aligned}
$$

The proof of the proposition is given in Appendix.

Using (4.28) and the proposition, we obtain

$$
\widehat{G}^{(0)}(z)=\widehat{\mathcal{G}} \cdot\left(1+O_{*}\left((m / W)^{3 / 2}\right)\right)^{-1}=\widehat{\mathcal{G}}+O_{*}\left((m / W)^{3 / 2}\right)
$$

This and (3.16) gives (4.10) (for $j>0$ the proof is similar).

To prove (4.11) for $K_{+}$, we use the representation of $K(\xi)$ in the form (3.4) - (3.6) (for $K_{-}$the proof is similar). Note that we have for $|\bar{k}| \leq m$ (cf. (3.29))

$$
\left(K \Psi_{\bar{k}, \delta}^{+}\right)(X)=O\left(e^{-C \log ^{2} W}\right), \quad \operatorname{dist}\left\{X, X_{+}\right\} \geq 2 W^{-1 / 2} \log W
$$

and so (similarly to the proof of Lemma 3.2) we can write for $\operatorname{dist}\left\{X, X_{+}\right\} \leq 2 W^{-1 / 2} \log W$, $\operatorname{dist}\left\{X, X_{+}\right\} \leq 2 W^{-1 / 2} \log W$

$$
\left(P_{+} A_{1} P_{+}\right)(X, Y)=A_{+, *}^{(m)}\left(x_{1}, x_{2}\right) A_{+, *}^{(m)}\left(y_{1}, y_{2}\right)\left(1+O_{*}\left((m / W)^{3 / 2}\right)\right) .
$$

Thus, to prove the first bound of (4.11), it suffices to prove that

$$
\left\|\left(A_{+, *}^{(m)} \otimes A_{+, *}^{(m)} \otimes A_{+, *}^{(m)} \otimes A_{+, *}^{(m)}-z\right)^{-1}\right\| \leq C W,
$$

which follows from (4.30) in the case, when the second condition of (4.29) is valid, and (3.16). 
Proof of Lemma 4.2. Using (3.53) and (4.26), by the same argumet as in Lemma 4.1, we get

$$
\begin{aligned}
& \left(P_{ \pm} K\left(I_{ \pm}-P_{ \pm}\right)\right)_{(\bar{k}, \bar{j}),\left(\bar{k}^{\prime}, \bar{j}^{\prime}\right)} \\
& =\delta_{\bar{j} \bar{j}^{\prime}} \lambda_{j}^{*} \cdot\left(\left(P_{+m} \otimes P_{-m}\right)\left(A^{+} \otimes A^{-}\right)\left(1-P_{+m} \otimes P_{-m}\right)\right)_{\bar{k}, \bar{k}^{\prime}}+\left(O_{*}\left((m / W)^{3 / 2}\right)\right)_{\bar{k}, \bar{k}^{\prime}} \\
& =\left(\sum _ { j \leq ( m W ) ^ { 1 / 2 } } \left(\lambda_{j}^{*} \cdot\left(P_{+m} \otimes P_{-m}\right)\left(A^{+} \otimes A^{-}\right)\left(1-P_{+m} \otimes P_{-m}\right)\right.\right. \\
& \left.\left.\quad+O_{*}\left((m / W)^{3 / 2}\right)\right) \otimes \mathcal{P}_{j}\right)_{(\bar{k}, \bar{j}),\left(\bar{k}^{\prime}, \bar{j}^{\prime}\right)},
\end{aligned}
$$

where $P_{+m}$ and $P_{-m}$ are defined by the same way as $P_{m}$ in Lemma 3.1 for the operators $A_{*}^{+}$ and $A_{*}^{-}$respectively. Hence, using that

$$
1-P_{+m} \otimes P_{-m}=P_{+m} \otimes\left(1-P_{-m}\right)+\left(1-P_{+m}\right) \otimes P_{-m}-\left(1-P_{+m}\right) \otimes\left(1-P_{-m}\right) .
$$

we obtain on the basis of Lemma 3.3 :

$$
\begin{gathered}
\quad\left\|P_{ \pm} K\left(I_{ \pm}-P_{ \pm}\right)\right\| \leq\left\|\left(P_{+m} \otimes P_{-m}\right)\left(A^{+} \otimes A^{-}\right)\left(1-P_{+m} \otimes P_{-m}\right)\right\|+O\left(m^{p} / W^{3 / 2}\right) \\
\leq\left\|\left(P_{+m} A^{+} P_{+m}\right) \otimes\left(P_{-m} A^{-}\left(1-P_{-m}\right)\right)\right\|+\left\|\left(P_{+m} A^{+}\left(1-P_{+m}\right)\right) \otimes\left(P_{-m} A^{-} P_{-m}\right)\right\| \\
\quad\left\|\left(P_{+m} A^{+}\left(1-P_{+m}\right)\right) \otimes\left(P_{-m} A^{-}\left(1-P_{-m}\right)\right)\right\|+O\left(m^{p} / W^{3 / 2}\right) \\
\leq C\left(\left\|P_{+m} A^{+}\left(1-P_{+m}\right)\right\|+\left\|P_{-m} A^{-}\left(1-P_{-m}\right)\right\|\right)+O\left(m^{p} / W^{3 / 2}\right) \leq C m W^{-1} .
\end{gathered}
$$

By the same way one can prove the bound for $\left(I_{ \pm}-P_{ \pm}\right) K P_{ \pm}, P_{+} K\left(I_{+}-P_{+}\right), P_{-} K\left(I_{-}-\right.$ $\left.P_{-}\right),\left(I_{+}-P_{+}\right) K P_{+}$, and $\left(I_{-}-P_{-}\right) K P_{-}$. The second line of (4.12) evidently follows from the first one combined with (3.37).

To prove (4.13), denote by $R_{j}$ the $j$ th operator in the r.h.s. of (4.31) (including the error term) and each $R_{j}$ split into two parts:

$$
\begin{aligned}
& R_{j}=R_{0 j}+R_{1 j}, \quad\left\|R_{1 j}\right\| \leq C(m / W)^{2}, \quad\left\|R_{0 j}\right\| \leq C(m / W), \\
& \left(R_{0 j} \Psi_{\bar{k}^{\prime}, j}, \Psi_{\bar{k}, j}\right)=0, \text { if }\left|\bar{k}^{\prime}\right|>m+3 .
\end{aligned}
$$

For this aim we set

$$
R_{1 j}=\lambda_{j}^{*}\left(\left(P_{+m} \otimes P_{-m}\right)\left(A^{+} \otimes A^{-}\right)\left(1-P_{+(m+3)} \otimes P_{-(m+3)}\right) \otimes \mathcal{P}_{j}\right)+O_{*}\left((m / W)^{3 / 2}\right),
$$

where $P_{+(m+3)}$ and $P_{-(m+3)}$ are defined as $P_{+m}$ and $P_{-m}$ with $m$ replaced by $m+3$. Then, using the same argument as above, we obtain the bound (4.32) for $\left\|R_{1 j}\right\|$. Setting $R_{0 j}=$ $R_{j}-R_{1 j}$, we obtain the second line of (4.32).

Note that by (4.6) and (4.31), to prove (4.13), it suffices to check the relations

$$
\begin{aligned}
& \left\|\left(K_{+}-z\right)^{-1} P_{+} K\left(I_{+}-P_{+}\right)\right\| \leq C m^{p}, \quad\left\|\left(K_{-}-z\right)^{-1} P_{-} K\left(I_{-}-P_{-}\right)\right\| \leq C m^{p}, \\
& \left\|\widehat{G}^{(0)} \widehat{R}_{0}\right\| \leq C m^{p}, \quad\left\|G^{(j)} R_{j}\right\| \leq C m^{p}, \quad j>1,
\end{aligned}
$$

with $R_{j}$, defined above, and $\widehat{R}_{0}$, being $R_{0}$ without the line, corresponding to $\Psi_{\overline{0}, 0, \delta}$.

The bounds of (4.33) follow from (4.11) and the second line of (4.12). The first bound of (4.34) follows from the first bound of (4.10) and (4.31). To obtain the second one, we use (4.32). The bounds of the norms of $R_{1 j}$ and $G^{(j)}$ yield

$$
\left\|G^{(j)} R_{1 j}\right\| \leq C m^{p}, \quad j>0 .
$$


Hence, to prove (4.13), we are left only to check that $\left\|G^{(j)} R_{0 j}\right\| \leq C m^{p}$.

By (3.16) and the bound for $F_{j}(z)$ from (4.10) it suffices to prove that if we denote by $R_{0 j}^{\left(\bar{k}^{\prime}\right)}$ the column of $R_{0 j}$ with a number $\bar{k}^{\prime}$, then

$$
\begin{aligned}
& \left|\left(\widehat{G}^{(j)} \kappa_{j}^{*}, R_{0 j}^{\left(\bar{k}^{\prime}\right)}\right)\right| \leq C m^{p} / W^{2}, \\
& \text { for } \bar{k}^{\prime}=\left(m+\alpha, k_{2}^{\prime}\right) \vee \bar{k}^{\prime}=\left(k_{1}^{\prime}, m+\alpha\right), \quad\left(\alpha=1,2,3, k_{1}^{\prime}, k_{2}^{\prime} \leq m\right) .
\end{aligned}
$$

Consider the case $k^{\prime}=\left(m+1, k_{2}^{\prime}\right)$ (other ones are similar). Using that $|\bar{l}| \geq\left(\left|l_{1}\right|+\left|l_{2}\right|\right) / 2$, we get

$$
\begin{aligned}
& \left|\left(R_{0 j}^{\left(\bar{k}^{\prime}\right)}\right)_{\bar{k}}\right|=\left|\lambda_{j}^{*} \cdot\left(A_{+}^{(m)}\right)_{k_{1}, m+1^{\prime}}\left(A_{-}^{(m)}\right)_{k_{2} k_{2}^{\prime}}\right|+O\left((C m / W)^{\left(m+1-k_{1}\right) / 4}\right) \\
& \leq\left.(C m / W)\right|^{\left(\left(m+1-k_{1}\right)+\left|k_{2}-k_{2}^{\prime}\right|\right) / 4} .
\end{aligned}
$$

By the definition (3.15) and the bounds (3.37), we have

$$
\left|\left(\kappa_{j}^{*}\right)_{\bar{k}}\right|=\left|\lambda_{j}^{*} \cdot\left(A_{+}\right)_{0 k_{1}}\left(A_{-}\right)_{0 k_{2}}\right|+O\left((C m / W)^{\left(k_{1}+k_{2}\right) / 4}\right) \leq(C m / W)^{\left(k_{1}+k_{2}\right) / 4} .
$$

Now, using (4.10), it is easy to obtain that

$$
\left|\left(\widehat{G}^{(j)} \kappa_{j}^{*}, R_{0 j}^{\left(\bar{k}^{\prime}\right)}\right)\right| \leq C W^{2} m^{p} q^{m / 4} \ll W^{-2}
$$

To estimate $\left\|G^{(j)} R_{1 j}\right\|$, one can just sum the bounds for different $k_{2}^{\prime} \leq m$ and add similar bounds for the other cases of (4.35). Thus, we proved (4.35) and hence finished the proof of (4.13).

Proof of Lemma 4.3. Let us split the integration domain $X \in \mathcal{H}$ into 3 sub domains, according to the value of the function $\mathcal{F}(X)$ (cf. (3.41)):

$$
\begin{aligned}
& \Lambda_{1}=\{X:|\mathcal{F}(X)| \geq 1-\delta / 2\}, \\
& \Lambda_{2}=\{X: 1-\delta / 2>|\mathcal{F}(X)| \geq 1-\delta\}, \\
& \Lambda_{3}=\{X: 1-\delta>|\mathcal{F}(X)|\},
\end{aligned}
$$

Then, similarly to the proof of Lemma 3.4, write

$$
u(X)=u_{1}(X)+u_{2}(X)+u_{3}(X)
$$

where $u_{i}(X)=u(X) 1_{X \in \Lambda_{i}}$. Since $\max _{X \in \Lambda_{2} \cup \Lambda_{3}}|\mathcal{F}(X)|=1-\delta$, we have similarly to (3.42) for any $u:\|u\|=1$

$$
\left\|u_{2}+u_{3}\right\|^{2} \leq C_{0}\left(1-\|K u\|^{2}\right)
$$

Moreover, similarly to (3.46)

$$
\Re\left(K\left(u_{1}+u_{2}\right), K u_{3}\right) \leq O\left(e^{-c W^{2}}\right)+\frac{1}{2}\left\|u_{2}+u_{3}\right\|^{2} .
$$

Hence, if we denote

$$
u_{0}:=u_{1}+u_{2}, \quad u_{0}^{( \pm)}=u_{0} 1_{\Omega_{\delta}^{( \pm)}}, \quad u_{0}^{(+)}=u_{0} 1_{\Omega_{\delta}^{(+)}}, \quad u_{0}^{(-)}=u_{0} 1_{\Omega_{\delta}^{(-)}},
$$

and prove for $u_{0}^{( \pm)}, u_{0}^{(+)}$, and $u_{0}^{(-)}$the analogue of Lemma 3.5, then repeating the bounds of (3.47)-(3.48), we obtain the bound for $K^{(22)}$. 
Lemma 4.4 For $u_{0}^{( \pm)}, u_{0}^{(+)}$, and $u_{0}^{(-)}$defined in 4.39) we have

$$
\begin{aligned}
& \left\|K u_{0}^{( \pm)}\right\|^{2} \leq 1-C m^{1 / 3} / W \\
& \left\|K u_{0}^{(-)}\right\|^{2} \leq 1-C m^{1 / 3} / W, \quad\left\|K u_{0}^{(+)}\right\|^{2} \leq 1-C m^{1 / 3} / W .
\end{aligned}
$$

Proof. To prove the first bound, let us note first that, by the assumption of the lemma,

$$
u_{0}^{( \pm)}=u_{01}^{( \pm)}+u_{02}^{( \pm)}+O\left(e^{-c W}\right)
$$

where

$$
u_{01}^{( \pm)}=\sum_{j \leq(m W)^{1 / 2}} \sum_{|\bar{k}|>m} u_{\bar{k}, j} \Psi_{\bar{k}, j}, \quad u_{02}^{( \pm)}=\sum_{j>(m W)^{1 / 2}} \sum_{\bar{k}} u_{\bar{k}, j} \Psi_{\bar{k}, j} .
$$

Hence,

$$
\left(K u_{01}^{( \pm)}, K u_{02}^{( \pm)}\right)_{p}=0 \Rightarrow\left\|K u_{01}^{( \pm)}+K u_{02}^{( \pm)}\right\|^{2}=\left\|K u_{01}^{( \pm)}\right\|^{2}+\left\|K u_{02}^{( \pm)}\right\|^{2} .
$$

Moreover, it is easy to see that the space $\mathcal{L}$ spanned on the functions $\left\{\Psi_{\bar{k}}(a, b) \phi_{j}(U)\right\}_{\bar{j}>(m W)^{1 / 2}}$ is invariant with respect to $K$ and for $u \in \mathcal{L}$

$$
\|K u\|^{2} \leq \max _{j>(m W)^{1 / 2}}\left(\lambda_{j} u, \lambda_{j} u\right)_{p} \leq\left(1-C m^{2} / W\right)\|u\|^{2}
$$

Thus

$$
\left\|K u_{02}^{( \pm)}\right\|^{2} \leq\left(1-C m^{2} / W\right)\left\|u_{02}^{( \pm)}\right\|^{2}
$$

To obtain a similar bound for $\left\|K u_{01}^{( \pm)}\right\|^{2}$, we use the same method, as in the proof of Lemma 3.5. Consider the operator kernel $A_{0,+}\left(a_{1}, a_{2}\right):=A_{0}\left(a_{1}, a_{2}\right)$, defined as in the proof of Lemma 3.5. and similarly define $A_{0,-}\left(a_{1}, a_{2}\right)$ (with $a_{-}$instead of $a_{+}$). Set

$$
K_{0}\left(a_{1}, a_{2}, b_{1}, b_{2}, U_{1}, U_{2}\right)=t^{-1} A_{0,+}\left(a_{1}, a_{2}\right) A_{0,-}\left(b_{1}, b_{2}\right) K_{*}\left(t, U_{1}, U_{2}\right)
$$

Choose $\widetilde{\Psi}_{\bar{k}}(a, b)=(a-b)^{-1} \widetilde{\psi}_{k_{1}}\left(a-a_{+}\right) \widetilde{\psi}_{k_{2}}\left(b-a_{-}\right), \bar{k}=\left(k_{1}, k_{2}\right)$. Then evidently

$$
\left(\widetilde{\Psi}_{\bar{k}}, \Psi_{\bar{k}^{\prime}}\right)_{p}=\left(\widetilde{\psi}_{k_{1}}, \psi_{k_{1}^{\prime}}\right)\left(\widetilde{\psi}_{k_{2}}, \psi_{k_{2}^{\prime}}\right) .
$$

By our choice of $u_{01}^{( \pm)}$

$$
u_{01}^{( \pm)}=\sum_{|\bar{k}| \geq m} u_{\bar{k}}(U) \Psi_{\bar{k}}+O\left(e^{-c W}\right) .
$$

Hence, setting $l=\left[m^{1 / 3}\right] / 3 C^{1 / 3}$ (with $C$ of (3.21) ) and denote by $\widetilde{P}_{l}$ the orthogonal projection operator on the linear span of $\left\{\widetilde{\Psi}_{\bar{k}}\right\}_{|\bar{k}| \leq l}$, we get

$$
\begin{aligned}
& \left\|\widetilde{P}_{l} u_{01}^{( \pm)}\right\|^{2} \leq \int d U \sum_{|\bar{k}| \leq l}\left|\left(u_{01}^{( \pm)}, \widetilde{\Psi}_{\bar{k}}\right)_{p}\right|^{2} \\
& =\int d U \sum_{|\bar{k}| \leq l}\left|\left(\sum_{k_{1}^{\prime} \geq m, k_{2}^{\prime}}+\sum_{k_{2}^{\prime} \geq m, k_{1}^{\prime}}-\sum_{k_{1}^{\prime}, k_{2}^{\prime} \geq m}\right) u_{\bar{k}^{\prime}}(U)\left(\psi_{k_{1}^{\prime}}, \tilde{\psi}_{k_{1}}\right)\left(\psi_{k_{2}^{\prime}}, \tilde{\psi}_{k_{2}}\right)\right|^{2} .
\end{aligned}
$$


Let us show how to estimate the first sum with respect to $\bar{k}^{\prime}$ above. Denote

$$
v_{k_{1}^{\prime}}=\sum_{k_{2}^{\prime}} u_{\bar{k}^{\prime}} \psi_{k_{2}^{\prime}} .
$$

Then, by the Schwartz inequality and (5.2) (see below), we obtain

$$
\begin{aligned}
\Sigma_{1}= & \int d U \sum_{k_{1}, k_{2} \leq l}\left|\sum_{k_{1}^{\prime}>m}\left(v_{k_{1}^{\prime}}, \tilde{\psi}_{k_{2}}\right)\left(\psi_{k_{1}^{\prime}}, \tilde{\psi}_{k_{1}}\right)\right|^{2} \\
& \leq \int d U\left(\sum_{k_{2} \leq l} \sum_{k_{1}^{\prime}>m}\left|\left(v_{k_{1}^{\prime}}, \tilde{\psi}_{k_{2}}\right)\right|^{2}\right)\left(\sum_{k_{1} \leq l} \sum_{k_{1}^{\prime}>m}\left|\left(\psi_{k_{1}^{\prime}}, \tilde{\psi}_{k_{1}}\right)\right|^{2}\right) \\
& \leq \tilde{C} \frac{l^{3}}{m} \sum_{k_{1}^{\prime}} \int d U\left(v_{k_{1}^{\prime}}, v_{k_{1}^{\prime}}\right) \leq \tilde{C} \frac{l^{3}}{m}\left\|u^{( \pm)}\right\|^{2} \leq\left\|u^{( \pm)}\right\|^{2} / 27 .
\end{aligned}
$$

Using similar bounds for the second and the third sum of (4.42) and denoting the respective expression by $\Sigma_{2}$ and $\Sigma_{3}$, we get

$$
\left\|\widetilde{P}_{l} u_{01}^{( \pm)}\right\|^{2} \leq 3 \Sigma_{1}+3 \Sigma_{2}+3 \Sigma_{3} \leq\left\|u^{( \pm)}\right\|^{2} / 3<\left\|u^{( \pm)}\right\|^{2} / 2
$$

Then, repeating the argument of Lemma 3.5, we get that

$$
\left\|K u_{01}^{( \pm)}\right\|^{2} \leq\left(1-C m^{1 / 3} / W\right)\left\|u_{01}^{( \pm)}\right\|^{2}
$$

and finish the proof of the lemma for $u_{0}^{( \pm)}$. For $u_{0}^{(+)}$and $u_{0}^{(-)}$the proofs are similar.

\section{Appendix}

Proof of Lemma 3.1. Orthonormality of $\left\{\psi_{k}\right\}_{k \geq 0}$ follows from the orthonormality of $\left\{p_{k}\right\}_{k \geq 0}$; (3.18) can be easily checked by the straightforward calculations.

Let us compute

$$
\begin{aligned}
A_{*}^{\left(c_{*}\right)} \psi_{k} & =\frac{h_{k}^{-1 / 2} W}{\sqrt{2 \pi}} \int e^{-W^{2}(x-y)^{2} / 2-c_{*}\left(x^{2}+y^{2}\right) / 2} \cdot e^{-\alpha W y^{2}} e^{2 \alpha_{1} W y^{2}}\left(\frac{d}{d y}\right)^{k} e^{-2 \alpha_{1} W y^{2}} d y \\
& =\frac{h_{k}^{-1 / 2} W}{\sqrt{2 \pi}} e^{W^{2} x^{2} / 2 d-\left(W^{2}+c_{*}^{2}\right) x^{2} / 2} \int e^{-W^{2} d(y-x / d)^{2} / 2}\left(\frac{d}{d y}\right)^{k} e^{-2 \alpha_{1} W y^{2}} d y,
\end{aligned}
$$

where

$$
d=1+c_{*} / W^{2}-2 \bar{\alpha} / W
$$

Integration by parts gives

$$
\begin{aligned}
A_{*}^{\left(c_{*}\right)} \psi_{k} & =\frac{(-1)^{k} h_{k}^{-1 / 2} W}{\sqrt{2 \pi}} e^{W^{2} x^{2} / 2 d-\left(W^{2}+c_{*}^{2}\right) x^{2} / 2} \int\left(\frac{d}{d y}\right)^{k} e^{-W^{2} d(y-x / d)^{2} / 2} e^{-2 \alpha_{1} W y^{2}} d y \\
& =\frac{d^{k} h_{k}^{-1 / 2} W}{\sqrt{2 \pi}} e^{W^{2} x^{2} / 2 d-\left(W^{2}+c_{*}^{2}\right) x^{2} / 2}\left(\frac{d}{d x}\right)^{k} \int e^{-W^{2} d(y-x / d)^{2} / 2-2 \alpha_{1} W y^{2}} d y
\end{aligned}
$$


and then we obtain by the straightforward calculations

$$
\begin{aligned}
& A_{*}^{\left(c_{*}\right)} \psi_{k}=h_{k}^{-1 / 2} \cdot \lambda_{0}^{\left(c_{*}\right)} \cdot\left(1-\frac{2 \bar{\alpha}}{W}+\frac{c_{*}}{W^{2}}\right)^{k} \cdot e^{d_{2} W x^{2}}\left(\frac{d}{d x}\right)^{k} e^{-d_{1} W x^{2}}, \\
& d_{1}=\frac{2 \alpha_{1}}{\left(1-\frac{2 \bar{\alpha}}{W}+\frac{c_{*}}{W^{2}}\right)\left(1+\frac{2 \alpha}{W}+\frac{c_{*}}{W^{2}}\right)}=2 \alpha_{1}\left(1-\frac{4 i \alpha_{2}}{W}\right)+O\left(W^{-2}\right), \\
& d_{2}=d_{1}-\alpha .
\end{aligned}
$$

According to (5.1), we have

$$
\begin{aligned}
& A_{*}^{\left(c_{*}\right)} \psi_{k}=\widetilde{p}_{k}(x) e^{-\alpha W x^{2}}, \quad \widetilde{p}_{k}(x)=\gamma_{k}^{(k)} x^{k}+\gamma_{k-2}^{(k)} x^{k-2}+\ldots, \\
& \gamma_{k}^{(k)}=\lambda_{0}^{\left(c_{*}\right)} \cdot h_{k}^{-1 / 2}\left(-2 d_{1} W\right)^{k} d^{k}, \quad \gamma_{k-2}^{(k)}=\frac{k(k-1)}{2} \cdot \lambda_{0}^{\left(c_{*}\right)} \cdot h_{k}^{-1 / 2}\left(-2 d_{1} W\right)^{k-1} d^{k} .
\end{aligned}
$$

Moreover, we can obtain from orthonormality of $\psi_{k}$

$$
\begin{aligned}
& \int x^{l} p_{k}(x) e^{-2 \alpha_{1} W x^{2}} d x=0, \quad l<k \\
& \int x^{k} p_{k}(x) e^{-2 \alpha_{1} W x^{2}} d x=h_{k}^{1 / 2}\left(-4 \alpha_{1} W\right)^{-k} \\
& \int x^{k+2} p_{k}(x) e^{-2 \alpha_{1} W x^{2}} d x=-h_{k}^{1 / 2}\left(-4 \alpha_{1} W\right)^{-k-1} \cdot \frac{(k+2)(k+1)}{2} .
\end{aligned}
$$

Hence,

$$
\begin{aligned}
\left(A_{*}^{\left(c_{*}\right)} \psi_{k}, \psi_{k}\right) & =\int \widetilde{p}_{k}(x) p_{k}(x) e^{-2 \alpha_{1} W x^{2}}=\int \gamma_{k}^{(k)} x^{k} \cdot p_{k}(x) e^{-2 \alpha_{1} W x^{2}} d x \\
& =d^{k}\left(d_{1} / 2 \alpha_{1}\right)^{k} \lambda_{0}^{\left(c_{*}\right)}=\left(\lambda_{0}^{\left(c_{*}\right)}\right)^{2 k+1}
\end{aligned}
$$

Similarly, we can write

$$
\begin{aligned}
\left(A_{*}^{\left(c_{*}\right)} \psi_{k+2}, \psi_{k}\right) & =\int \widetilde{p}_{k+2}(x) p_{k}(x) e^{-2 \alpha_{1} W x^{2}} d x \\
& =\int\left(\gamma_{k+2}^{(k+2)} x^{k+2}+\gamma_{k}^{(k+2)} x^{k}\right) p_{k}(x) e^{-2 \alpha_{1} W x^{2}} d x \\
& =\frac{\sqrt{(k+2)(k+1)}}{2}\left(\frac{d_{1}}{2 \alpha_{1}}\right)^{k+1}\left(\frac{d_{1}}{2 \alpha_{1}}-1\right) \cdot \lambda_{0}^{\left(c_{*}\right)} \cdot d^{k+2},
\end{aligned}
$$

which gives (3.19). By the same argument one can obtain (3.20), using that

$$
\frac{\sqrt{(k+1)(k+2) \ldots(k+2 l)}}{l !} \leq C^{l}(k+1)^{l} .
$$

To prove (3.21), let us prove first that for $j, k>2$

$$
\left|\left(\psi_{j}, \widetilde{\psi}_{k}\right)\right| \leq C(k / j)
$$

Indeed, changing $x \rightarrow x / \sqrt{W}$, we get

$$
\left(\psi_{j}, \widetilde{\psi}_{k}\right)=\left(\varphi_{j}, e \widetilde{\varphi}_{k}\right)
$$


where

$$
\begin{aligned}
\varphi_{j}(x) & =h_{j 0}^{-1 / 2}\left(\frac{d}{d x}\right)^{j} e^{-2 \alpha_{1} x^{2}}, \quad h_{j 0}=j !\left(4 \alpha_{1}\right)^{j-1 / 2} \sqrt{2 \pi}, \\
\widetilde{\varphi}_{k}(x) & =\widetilde{h}_{k 0}^{-1 / 2}\left(\frac{d}{d x}\right)^{k} e^{-2 \widetilde{\alpha} x^{2}}, \quad \widetilde{h}_{k 0}=k !(4 \widetilde{\alpha})^{k-1 / 2} \sqrt{2 \pi}, \\
e(x) & =e^{(\bar{\alpha}+\widetilde{\alpha}) x^{2}} .
\end{aligned}
$$

Since

$$
\varphi_{j}(x)=\left(4 \alpha_{1}\right)^{-1}(j(j-1))^{-1 / 2} \varphi_{j-2}^{\prime \prime}(x),
$$

and similar relations are valid for $\widetilde{\varphi}_{k}(x)$, integration by parts yelds

$$
\begin{aligned}
&\left(\varphi_{j}, e \widetilde{\varphi}_{k}\right)=\left(4 \alpha_{1}\right)^{-1}(j(j-1))^{-1 / 2}\left(\varphi_{j-2},\left(e \varphi_{k}\right)^{\prime \prime}\right) \\
&=\left(4 \alpha_{1}\right)^{-1}(j(j-1))^{-1 / 2}\left(\varphi_{j-2}, e^{\prime \prime} \widetilde{\varphi}_{k}+2 e^{\prime}(\alpha(k+1))^{1 / 2} \varphi_{k+1}\right. \\
&\left.\quad+e \cdot 4 \widetilde{\alpha}((k+2)(k+1))^{1 / 2} 4 \widetilde{\alpha} \varphi_{k+2}\right) .
\end{aligned}
$$

Since $\varphi_{k} e^{\alpha_{1} x^{2}}$ and $\widetilde{\varphi}_{j} e^{\widetilde{\alpha} x^{2}}$ by definition (5.3) are the normalized Hermite functions, the above relation proves (5.2). Now, let $u=\left(1-P_{m}\right) u$, hence

$$
u=\sum_{k>m} u_{k} \psi_{k}
$$

Then, by (5.2),

$$
\begin{aligned}
& \left\|P_{l} u\right\|^{2}=\sum_{k=0}^{l}\left|\left(u, \widetilde{\psi}_{k}\right)\right|^{2}=\sum_{k=0}^{l}\left|\sum_{j>m} u_{j}\left(\psi_{j}, \widetilde{\psi}_{k}\right)\right|^{2} \leq\|u\|^{2} \sum_{k=0}^{l} \sum_{j>m}\left|\left(\psi_{j}, \widetilde{\psi}_{k}\right)\right|^{2} \\
& \leq C\|u\|^{2} \sum_{k \leq l} \sum_{j>m} \frac{k^{2}}{j^{2}} \leq \frac{C\|u\|^{2}}{m} \sum_{k \leq l} k^{2} \leq C\|u\|^{2} \frac{l^{3}}{m}
\end{aligned}
$$

Proof of Proposition 4.1. Similarly to the proof of Lemma 3.2, consider the diagonal matrix with the entries

$$
D_{\bar{k} \bar{k}}=A_{* k_{1} k_{1}}^{\sigma_{1}} \ldots A_{* k_{s} k_{s}}^{\sigma_{s}}-z
$$

As in (3.35), we have

$$
\left|D_{\bar{k} \bar{k}}\right|>2 \alpha_{1}\left(k_{1}+\cdots+k_{s}+s / 2-\varepsilon\right) / W .
$$

Set also $R=\left(\widehat{K}_{ \pm}^{(0)}-D-z\right) D^{-1}$ and let $Q$ be the matrix which contains $O(1)$-order entries of $R$ while the other ones are replaced by zeros. This gives

$$
\left(\widehat{K}_{ \pm}^{(0)}-z\right)^{-1}=D^{-1}(I+R)^{-1}=D^{-1}(1+Q)^{-1}(I+\widetilde{R})^{-1}
$$

where $\widetilde{R}=(R-Q)(I+Q)^{-1}$.

It follows from (3.37) that, if we set $e_{1}=(1,0, \ldots, 0), \ldots e_{s}=(0, \ldots, 0,1)$, then

$$
Q_{\bar{k} \bar{k}^{\prime}} \neq 0, \text { iff } \bar{k}^{\prime}=\bar{k}+2 e_{1} \vee \cdots \vee \bar{k}^{\prime}=\bar{k}+2 e_{s}
$$


Moreover, there exists an absolute constant $l_{\alpha}$, such that for $|k|>l_{\alpha}$

$$
\begin{aligned}
& \sum_{i=1}^{s}\left|Q_{\bar{k}, \bar{k}+2 e_{i}}\right| \leq \sum_{i=1}^{s} \frac{\left|A_{* k_{i}, k_{i}+2}^{\sigma_{i}}\right|}{2 \alpha_{1}\left(k_{1}+\cdots+k_{s}+s / 2\right)} \\
\leq & \frac{\alpha_{2}}{\alpha_{1}} \frac{\sqrt{\left(k_{1}+1\right)\left(k_{1}+2\right)}+\cdots+\sqrt{\left(k_{s}+1\right)\left(k_{s}+2\right)}}{\left(k_{1}+\cdots+k_{s}+s / 2-\varepsilon\right)} \leq\left(\alpha_{2} / \alpha_{1}\right)^{1 / 2}=q<1 .
\end{aligned}
$$

Here we used that $\alpha_{2}<\alpha_{1}$ (see (3.36) $)$.

Write $Q$ as a block matrix

$$
\begin{aligned}
& Q^{(11)}=\left\{Q_{\bar{k}, \bar{k}^{\prime}}\right\}_{|k| \leq l_{\alpha},\left|k^{\prime}\right| \leq l_{\alpha},} Q^{(12)}=\left\{Q_{\bar{k}, \bar{k}^{\prime}}\right\}_{|k| \leq l_{\alpha},\left|k^{\prime}\right|>l_{\alpha}}, \\
& Q^{(21)}=\left\{Q_{\bar{k}, \bar{k}^{\prime}}\right\}_{|k|>l_{\alpha},\left|k^{\prime}\right| \leq l_{\alpha}} \quad Q^{(22)}=\left\{Q_{\bar{k}, \bar{k}^{\prime}}\right\}_{|k|>l_{\alpha},\left|k^{\prime}\right|>l_{\alpha}} .
\end{aligned}
$$

Then by (15.6) $Q^{(21)}=0$, and by (3.8) $\left\|Q^{(22)}\right\| \leq q$. Moreover, (5.6) implies that for $s_{0}=\left[l_{\alpha} / 2\right]+1$

$$
Q^{s_{0}}=\left(\begin{array}{cc}
0 & X \\
0 & \left(Q^{(22)}\right)^{s_{0}}
\end{array}\right) \Rightarrow Q^{s_{0}+p}=\left(\begin{array}{cc}
0 & X\left(Q^{(22)}\right)^{p} \\
0 & \left(Q^{(22)}\right)^{s_{0}+p}
\end{array}\right), \quad p>0
$$

where $X$ is some fixed matrix. Writing the Neumann series $(1+Q)^{-1}=\sum_{s}(-1)^{s} Q^{s}$ and taking into account that by (5.6) $)\left(Q^{s}\right)_{\bar{k} \bar{k}^{\prime}}=0$, till $s<\left|\bar{k}-\bar{k}^{\prime}\right| / 2$, we obtain that

$$
\left|(1+Q)_{\bar{k} \bar{k}^{\prime}}^{-1}\right| \leq C q^{\left|\bar{k}-\bar{k}^{\prime}\right| / 2}
$$

Besides, it follows from (3.37) that $R-Q=O_{*}\left((m / W)^{3 / 2}\right)$, hence

$$
\left|(\widetilde{R})_{\bar{k} \bar{k}^{\prime}}\right|=\left|\sum_{\mid \bar{k}^{\prime \prime} \leq m}(R-Q)_{\bar{k} \bar{k}^{\prime \prime}}(1+Q)_{\bar{k}^{\prime \prime}, \bar{k}^{\prime}}\right| \leq C\left(m^{5 / 2} / W^{-1 / 2}\right) q^{\left|\bar{k}-\bar{k}^{\prime}\right| / 2},
$$

The last relation implies

$$
\left|(1+\widetilde{R})_{\bar{k} \bar{k}^{\prime}}^{-1}\right| \leq C q^{-\left|\bar{k}-\bar{k}^{\prime}\right| / 2} .
$$

To prove this, let us consider any fixed $\bar{k}$ and $\bar{k}^{\prime}$ and use the standard trick from the spectral theory (see e.g. [14, Ch. 13.3). Assume that $\left|\bar{k}-\bar{k}^{\prime}\right|=k_{1}-k_{1}^{\prime}$. Then denote $D_{q}$ the diagonal matrix such that $\left(D_{q}\right)_{\bar{k}^{\prime \prime} \bar{k}^{\prime \prime \prime}}=\delta_{\bar{k}^{\prime \prime} \bar{k}^{\prime \prime \prime}} q^{k_{1}^{\prime \prime} / 2}$. Then

$$
\begin{aligned}
& \quad|| D_{q} \widetilde{R} D_{q}^{-1} \| \leq C m^{7 / 2} / W^{-1 / 2} \\
& \Rightarrow\left|(1-\widetilde{R})_{\bar{k} \bar{k}^{\prime}}^{-1}\right|=\left|\left(D_{q}\left(1-D_{q} \widetilde{R} D_{q}^{-1}\right)^{-1} D_{q}^{-1}\right)_{\bar{k} \bar{k}^{\prime}}\right| \\
& \quad \leq q^{\left(k_{1}-k_{1}^{\prime}\right) / 2}||\left(1-D_{q} \widetilde{R} D_{q}^{-1}\right)^{-1} \| .
\end{aligned}
$$

If $\left|\bar{k}-\bar{k}^{\prime}\right|=-\left(k_{1}-k_{1}^{\prime}\right)$ we use $D_{q}^{-1}$ instead of $D_{q}$. And if $\left|\bar{k}-\bar{k}^{\prime}\right|= \pm\left(k_{2}-k_{2}^{\prime}\right)$ we use $\left(D_{q}\right)_{\bar{k}^{\prime \prime} \bar{k}^{\prime \prime \prime}}=\delta_{\bar{k}^{\prime \prime} \bar{k}^{\prime \prime \prime}} q^{ \pm k_{2}^{\prime \prime} / 2}$.

Now (5.7), (5.8), and (5.5) conclude the proof of the first line of (4.30). The second line follows from the fact that

$$
F\left(\lambda_{0}(\mathcal{A})\right)=0 \quad F^{\prime}(z)=1-\left(\widehat{\mathcal{G}}^{2} \kappa, \kappa^{*}\right)=1-O\left(m^{p} W^{-1 / 2}\right) .
$$


Proof of Proposition 3.2. Notice that, if for any $V \in \stackrel{\circ}{U}(2)$ we define an operator

$$
\left(T_{V} f\right)(U)=f(U V)
$$

then for any kernel of the form $\mathcal{K}\left(U_{1}, U_{2}\right)=\mathcal{K}\left(U_{1} U_{2}^{*}\right)$ we have evidently

$$
\begin{aligned}
& T_{V} \mathcal{K} f(U)=\int \mathcal{K}\left(U V U_{1}^{*}\right) f\left(U_{1}\right) d U_{1}=\int \mathcal{K}\left(U U_{2}^{*}\right) f\left(U_{2} V\right) d U_{2}=\mathcal{K} T_{V} f(U) \\
& \Rightarrow T_{V} \mathcal{K}=\mathcal{K} T_{V}
\end{aligned}
$$

Since $T_{V}$ is a representation of the group $\Theta^{U}(2)$ in $L_{2}\left[{ }^{U}(2), d U\right]$, it can be represented as a sum of irreducible representations in the subspaces $E_{j}\left(L_{2}[d U]=\oplus E_{j}\right)$. And the commutation property guarantees that $\mathcal{K}$ acts like an identity operator multiplied by some constant in each of the subspace $E_{j}$. These constants $\lambda_{j}$ are eigenvalues of $\mathcal{K}$, and choosing any basis in $E_{j}$ we obtain all eigenvectors of $\mathcal{K}$.

In the standard parametrization

$$
U=\left(\begin{array}{cc}
\cos \varphi & \sin \varphi \cdot e^{i \theta} \\
-\sin \varphi \cdot e^{-i \theta} & \cos \varphi
\end{array}\right)
$$

the measure $d U$ has the form

$$
d U=\frac{1}{\pi} u d u d \theta, \quad u=|\sin \varphi| \in[0,1], \quad \theta \in[0,2 \pi) .
$$

Then the spaces $E_{j}$ of the irreducible representations are well-known, and the proper basis in $E_{j}$ is made from the standard spherical harmonics $\phi_{\bar{j}}(U)$ with $\bar{j}=(j, k), j=0,1, \ldots$, $k=-j, \ldots, j$ be

$$
\phi_{\bar{j}}(U)=l_{j, k} P_{j}^{k}(\cos 2 \phi) e^{i k \theta}=\left.l_{j, k}\left(\frac{d}{d x}\right)^{k} P_{j}(x)\right|_{x=1-2\left|U_{12}\right|^{2}}\left(2 \bar{U}_{11} U_{12}\right)^{k},
$$

where $P_{j}^{k}$ is an associated Legendre polynomial

$$
\begin{aligned}
& P_{j}^{k}(\cos x)=(\sin x)^{k}\left(\frac{d}{d \cos x}\right)^{k} P_{j}(\cos x), \quad P_{j}(x)=\frac{1}{2^{j} j !} \frac{d^{j}}{d x^{j}}\left(x^{2}-1\right)^{j}, \\
& l_{j, k}=\sqrt{\frac{(2 j+1)(j-k) !}{(j+k) !}}
\end{aligned}
$$

Then $\left\{\phi_{\bar{j}}(U)\right\}$ is an orthonormal basis (see, e.g., [1, $\S 12.6$ ); to find $\lambda_{j}$ it suffices to apply our $K_{*}$ to $P_{j}$. We get

$$
\begin{array}{r}
\lambda_{j}(t)=\left(K_{*} \phi_{(j, 0)}, \phi_{(j, 0)}\right)=\int K_{*}\left(t, U_{1}, U_{2}\right) \phi_{(j, 0)}\left(U_{2}\right) \phi_{(j, 0)}\left(U_{1}\right) d U_{1} d U_{2} \\
=t W^{2} \int e^{-t W^{2}\left|\left(U_{1} U_{2}^{*}\right)_{12}\right|^{2}} f\left(U_{2}\right) \phi_{(j, 0)}\left(U_{2}\right) \phi_{(j, 0)}\left(U_{1}\right) d U_{1} d U_{2} \\
=t W^{2} \int e^{-t W^{2}\left|U_{12}\right|^{2}} \phi_{(j, 0)}\left(U_{1} U^{*}\right) \phi_{(j, 0)}\left(U_{1}\right) d U d U_{1}
\end{array}
$$


For the parametrization (5.9) we have

$$
\phi_{(j, 0)}(U)=P_{j}\left(1-2\left|U_{12}\right|^{2}\right) \Rightarrow \phi_{(j, 0)}\left(U_{1} U^{*}\right)=P_{j}\left(1-2\left|\left(U_{1} U^{*}\right)_{12}\right|^{2}\right) .
$$

Since the Legendre addition theorem (see, e.g., [1], §12.8) yields

$$
\begin{aligned}
P_{j}\left(1-2\left|\left(U_{1} U^{*}\right)_{12}\right|^{2}\right)=P_{j}( & \cos 2 \varphi) \cdot P_{j}\left(\cos 2 \varphi_{1}\right) \\
& +2 \sum_{l=1}^{j} \frac{(l-j) !}{(l+j) !} P_{j}^{l}(\cos 2 \varphi) \cdot P_{j}^{l}\left(\cos 2 \varphi_{1}\right) \cdot \cos \left(k\left(\theta-\theta_{1}\right)\right),
\end{aligned}
$$

integrating first with respect to $\theta_{1}$, we obtain that the sum above gives a zero contribution to the integral (5.12) and

$$
\int d U_{1} \phi_{(j, 0)}(U) \phi_{(j, 0)}\left(U_{1} U^{*}\right)=P_{j}(\cos 2 \phi)
$$

Thus,

$$
\lambda_{\bar{j}}(t)=2 t W^{2} \int_{0}^{1} e^{-t W^{2} u^{2}} P_{j}\left(1-2 u^{2}\right) u d u=\int_{0}^{t W^{2}} e^{-u} P_{j}\left(1-\frac{2 u}{t W^{2}}\right) d u
$$

which gives the first line of (3.52), since

$$
\begin{aligned}
\left.\frac{d^{l}}{d x^{l}} P_{j}(x)\right|_{x=1} & =\left.\frac{1}{2^{j} j !} \frac{d^{j+l}}{d x^{j+l}}\left(x^{2}-1\right)^{j}\right|_{x=1}=\frac{1}{2 \pi i} \cdot \frac{(j+l) !}{2^{j} j !} \oint \frac{\left(z^{2}-1\right)^{j}}{(z-1)^{j+l+1}} d z \\
& =\frac{(j+l)(j+l-1) \ldots(j-l+1)}{2^{l} l !} .
\end{aligned}
$$

The second line of (3.52) can be obtained easily from the direct computations.

Acknowledgement. We are grateful to Sasha Sodin, who drew our attention to the transfer matrix approach in application to $1 \mathrm{~d}$ random band matrices, for many fruitful discussion. Also we would like to thank Leonid Pastur for his helpful suggestions on the proof of Proposition 3.2, An essential part of this work was done during our stay at the Simons Center of Geometry and Physics, which we would like to thank for its hospitality.

\section{References}

[1] Arfken, G. Mathematical Methods for Physicists, 3rd ed. Orlando, FL: Academic Press, 1985.

[2] Bao, J., Erdös, L.: Delocalization for a class of random block band matrices, arXiv:1503.07510

[3] Bogachev, L. V., Molchanov, S. A., and Pastur, L. A.: On the level density of random band matrices. Mat. Zametki, 50:6, 31 - 42 (1991)

[4] Bourgade, P., Erdős, L., Yau, H.-T., Yin, J. Universality for a class of random band matrices, arXiv:1602.02312 
[5] Brézin, E., Hikami, S.: Characteristic polynomials of random matrices. Commun. Math. Phys. 214, $111-135$ (2000)

[6] Brézin, E., Hikami, S.: Characteristic polynomials of real symmetric random matrices. Commun. Math. Phys. 223, 363 - 382 (2001)

[7] Casati, G., Molinari, L., Israilev, F.: Scaling properties of band random matrices, Phys. Rev. Lett. 64 , $1851-1854$ (1990).

[8] Disertori, M., Sodin, S.: Semi-classical analysis of non self-adjoint transfer matrices in statistical mechanics. I, Annal. Henri Poincaré, http://dx.doi.org/10.1007/s00023-015-0397-x (2015)

[9] Erdős, L., Knowles, A.: Quantum diffusion and eigenfunction delocalization in a random band matrix model. Commun. Math. Phys. 303, 509 - 554 (2011).

[10] Erdős, L., Knowles, A., Yau, H.-T., Yin, J.: Delocalization and diffusion profile for random band matrices, Commun.Math.Phys. 323, 367 - 416 (2013).

[11] Erdős, L., Yau, H.-T., Yin, J.: Bulk universality for generalized Wigner matrices, Probab. Theory Relat. Fields 154, 341 - 407 (2012)

[12] Fyodorov, Y.V., Mirlin, A.D.: Scaling properties of localization in random band matrices: a $\sigma$-model approach, Phys. Rev. Lett. 67, 2405 - 2409 (1991).

[13] Molchanov, S. A., Pastur, L. A., Khorunzhii, A. M.: Distribution of the eigenvalues of random band matrices in the limit of their infinite order, Theor. Math. Phys. 90, 108 118 (1992)

[14] Pastur, L. A., Shcherbina, M. Eigenvalue distribution of large random matrices. American mathematical society, 2011.

[15] Schenker, J.: Eigenvector localization for random band matrices with power law band width, Commun. Math. Phys. 290, 1065 - 1097 (2009)

[16] Shcherbina, T.: On the correlation function of the characteristic polynomials of the hermitian Wigner ensemble. Commun. Math. Phys. 308, p. 1 - 21 (2011)

[17] Shcherbina, T.: On the correlation functions of the characteristic polynomials of the hermitian sample covariance ensemble, Probab. Theory Relat. Fields 156, p. 449 - 482 (2013)

[18] Shcherbina, T. : On the second mixed moment of the characteristic polynomials of the 1D band matrices. Commun. Math. Phys. 328, p. $45-82$ (2014)

[19] Shcherbina, T.: Universality of the local regime for the block band matrices with a finite number of blocks. J.Stat.Phys. 155, 3, p. 466 - 499 (2014)

[20] Tao, T., Vu, V.: Random matrices: Universality of the local eigenvalue statistics. Acta Math. 206, 127 - 204 (2011). 